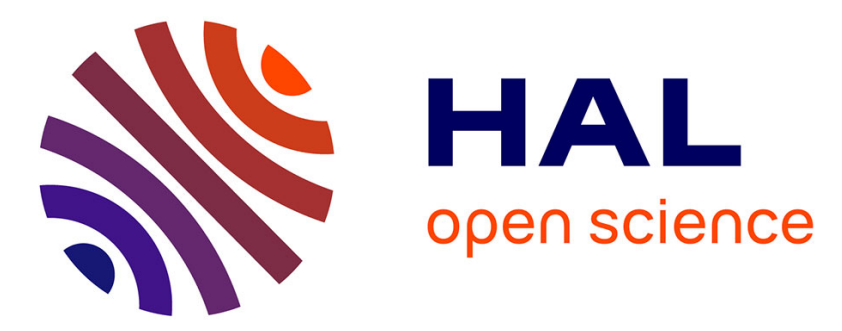

\title{
On the three-dimensional stationary exterior Stokes problem with Non Standard Boundary Conditions
}

Héla Louati, Mohamed Meslameni, Ulrich Razafison

\section{To cite this version:}

Héla Louati, Mohamed Meslameni, Ulrich Razafison. On the three-dimensional stationary exterior Stokes problem with Non Standard Boundary Conditions. Journal of Applied Mathematics and Mechanics / Zeitschrift für Angewandte Mathematik und Mechanik, 2020, 100 (6), 10.1002/zamm.201900181. hal-02174108v2

\section{HAL Id: hal-02174108 \\ https://hal.science/hal-02174108v2}

Submitted on 31 Jul 2020

HAL is a multi-disciplinary open access archive for the deposit and dissemination of scientific research documents, whether they are published or not. The documents may come from teaching and research institutions in France or abroad, or from public or private research centers.
L'archive ouverte pluridisciplinaire HAL, est destinée au dépôt et à la diffusion de documents scientifiques de niveau recherche, publiés ou non, émanant des établissements d'enseignement et de recherche français ou étrangers, des laboratoires publics ou privés. 


\title{
On the three-dimensional stationary exterior Stokes problem with Non Standard Boundary Conditions
}

\author{
Hela Louati ${ }^{1,2}$ \\ Mohamed Meslameni ${ }^{2,3}$ \\ Ulrich Razafison $^{4}$ \\ 1 - Faculty of Sciences of Sfax-Tunisia \\ University of Sfax, \\ Route de Soukra, km 3.5, BP 1171 \\ 3000 Sfax - Tunisia \\ 2 - College of Arts and Science in Al-Mandaq \\ AL Baha University - Kingdom of Saudi Arabia \\ 3 - Preparatory Institute for engineering Studies of Sfax \\ University of Sfax - Tunisia \\ 4 - Laboratoire de Mathématiques de Besançon \\ CNRS UMR 6623, \\ Université Bourgogne Franche-Comté, \\ 16 route de Gray, 25030 Besançon Cedex, France
}

\begin{abstract}
We study the three-dimensional stationary exterior Stokes problem with non standard boundary conditions corresponding to a slip-without-friction boundary conditions. Because the flow domain is unbounded, we set the problem in weighted spaces in order to control the behavior at infinity of solutions. This functional framework allows to prescribe various behaviors at infinity of the solutions. The results established are related to the existence and the uniqueness of strong and very weak solutions. Our strategy relies on the fact that due to the boundary conditions, the pressure and the velocity can be decoupled and, as a result, we solve two separate systems to find these quantities.
\end{abstract}

Keywords : Fluid mechanics, Stokes equations, Slip boundary conditions, Exterior domains, Strong solutions, Very weak solutions, Weighted spaces

AMS Classification: 76D07, 35J25, 76D03, 35J50

\section{Introduction}

Let $\Omega^{\prime}$ be a simply-connected bounded domain of $\mathbb{R}^{3}$ assumed to have a boundary $\Gamma$ of class $\mathscr{C}^{2,1}$ and let $\Omega$ denote the complement of $\overline{\Omega^{\prime}}$, in other words, the exterior of $\Omega^{\prime}$. For a prescribed external force $\boldsymbol{f}$, we 
consider the stationary Stokes equations, describing the flow of a viscous and incompressible fluid past the obstacle $\Omega^{\prime}$ :

$$
-\Delta \boldsymbol{u}+\nabla \pi=\boldsymbol{f} \text { and } \operatorname{div} \boldsymbol{u}=0 \text { in } \Omega,
$$

where the unknowns are the velocity field $\boldsymbol{u}$ and the pressure $\pi$. To these equations, we supplement the following Navier's type slip-without-friction boundary conditions:

$$
\boldsymbol{u} \cdot \boldsymbol{n}=0 \quad \text { and } \quad \operatorname{curl} \boldsymbol{u} \times \boldsymbol{n}=\mathbf{0} \quad \text { on } \quad \Gamma \text {, }
$$

where curl $\boldsymbol{u}$ is the vorticity field and $\boldsymbol{n}$ is the unit normal vector to the boundary $\Gamma$ pointing outside $\Omega$. As it is now well known, the boundary conditions (1.2) are closely related to the classical Navier boundary conditions, in which it is assumed that there is a stagnant layer of fluid close to the obstacle $\Omega^{\prime}$ allowing the fluid to slip and the slip velocity is proportional to the rate-of-strain tensor field:

$$
\boldsymbol{u} \cdot \boldsymbol{n}=0 \quad \text { and } \quad 2[\mathbf{D}(\boldsymbol{u}) \boldsymbol{n}]_{\boldsymbol{\tau}}+\alpha \boldsymbol{u}_{\boldsymbol{\tau}}=\mathbf{0} \quad \text { on } \quad \Gamma \text {, }
$$

where

$$
\mathbf{D}(\boldsymbol{u})=\frac{1}{2}\left(\nabla \boldsymbol{u}+\nabla \boldsymbol{u}^{T}\right)
$$

denotes the rate-of-strain tensor field, $\alpha$ is a scalar friction function and the notation $[\cdot]_{\boldsymbol{\tau}}$ denotes the tangential component of a vector on $\Gamma$. The boundary conditions (1.3), proposed by Navier [26] are commonly used in the presence of rough boundaries (see for instance [2, 21]). We also refer to [27] for a review of experimental studies that show various situations in which the slip property occurs.

The link between the boundary conditions (1.2) and (1.3) can be highlighted using, on the one hand, the fact that (1.2) is equivalent to (see [29])

$$
\boldsymbol{u} \cdot \boldsymbol{n}=0 \text { and } \nabla \boldsymbol{u}_{\boldsymbol{\tau}} \cdot \boldsymbol{n}=\mathbf{0} \text { on } \Gamma
$$

and on the other hand, the fact that the following identity holds (see [28])

$$
2[\mathbf{D}(\boldsymbol{u}) \boldsymbol{n}]_{\boldsymbol{\tau}}=\nabla \boldsymbol{u}_{\boldsymbol{\tau}} \cdot \boldsymbol{n}-k_{\boldsymbol{\tau}} \boldsymbol{u}_{\boldsymbol{\tau}}+[\nabla(\boldsymbol{u} \cdot \boldsymbol{n})]_{\boldsymbol{\tau}} \quad \text { on } \quad \Gamma,
$$

where $k_{\tau}$ is the principal curvature of $\Gamma$. Indeed we see that (1.2) is then equivalent to

$$
\boldsymbol{u} \cdot \boldsymbol{n}=0 \quad \text { and } 2[\mathbf{D}(\boldsymbol{u}) \boldsymbol{n}]_{\boldsymbol{\tau}}+k_{\boldsymbol{\tau}} \boldsymbol{u}_{\boldsymbol{\tau}}=\mathbf{0} \quad \text { on } \quad \Gamma,
$$

that is the Navier boundary condition (1.3) where the friction function is replaced by the principal curvature of $\Gamma$. Moreover, we also see that on flat boundaries, the boundary conditions (1.2) coincide exactly with the Navier boundary conditions (1.3) without friction. It is therefore interesting to study the exterior Stokes problem (1.1) with the boundary conditions (1.2). For further discussions on the relationships be- 
tween the boundary conditions (1.2) and (1.3), the reader can also refer to [14] or [25] and references therein.

The objective of this paper is to investigate the existence and the uniqueness of strong and very weak solution of the exterior problem (1.1)-(1.2). Because the domain is unbounded, the data and the solutions are assumed to belong to adequate weighted Sobolev spaces in order to control and to precise the behaviour of the functions at infinity. The main contribution of the paper is that the results are established for a very wide range of behaviour at infinity (growth or decay at infinity). This work follows a previous paper of one of the authors [5] in which a solution with a particular behaviour at infinity, corresponding to the variational solution and its regularity, was investigated.

Moreover, this work is also a starting point for the numerical analysis of (1.1)-(1.2), using the so-called inverted finite elements method originally developed by Boulmezaoud in [8] (see also, e.g., [7, 9, 10]) where the use of weighted Sobolev spaces is in the heart of the method.

In order to study (1.1)-(1.2), our strategy is the following: due to the boundary conditions (1.2), we can look for the pressure $\pi$ separately from the velocity $\boldsymbol{u}$. This can be done by solving, on the one hand, the generalized Neumann problem

$$
\operatorname{div}(\nabla \pi-\boldsymbol{f})=0 \quad \text { in } \quad \Omega \quad \text { and } \quad(\nabla \pi-\boldsymbol{f}) \cdot \boldsymbol{n}=0 \quad \text { on } \quad \Gamma \text {, }
$$

and on the other hand, the so-called Hodge-Laplacian problem

$$
\left\{\begin{aligned}
-\Delta \boldsymbol{u}=\boldsymbol{F} & \text { and } \operatorname{div} \boldsymbol{u}=0 \quad \text { in } \quad \Omega, \\
\boldsymbol{u} \cdot \boldsymbol{n}=0 & \text { and } \quad \operatorname{curl} \boldsymbol{u} \times \boldsymbol{n}=\mathbf{0} \text { on } \Gamma,
\end{aligned}\right.
$$

where $\boldsymbol{F}=\boldsymbol{f}-\nabla \pi$. This gives the opportunity to solve the exterior problems (1.6) and (1.7) that are also interesting by themselves. Let us emphasize that solving these problems is not straightforward because we are really interested in various behaviour at infinity of the solutions. Indeed, the method that we use to solve (1.6) and (1.7), relies on the properties of the Laplace operator in the whole space $\mathbb{R}^{3}$. But these properties naturally bring out compatibility conditions that the data necessarily satisfy when looking for solutions that decay faster than the variational solution. As a result, the solutions defined on $\Omega$ that we already have in hand (generally the variational solution) must be extended to the whole space in an appropriate manner in order to satisfy the compatibility conditions. Observe that these difficulties can be avoided when studying the standard exterior Laplace problem with Dirichlet boundary conditions, by the use of duality arguments (see, e.g. [18]). But this approach can not be used for problem (1.7) due to the fact that the Hodge-Laplacian operator is not a self adjoint operator.

Observe also that, in [5], the study of (1.1)-(1.2) was also based on the resolution of (1.7). But, because the study was focusing on the variational solution, then using the variational formulation, the pressure $\pi$ was obtained thanks to de Rham theorem and characterizations of distributions by means of their gradients. 
We would like to mention that, to the best of our knowledge, the exterior problem (1.1)-(1.2) has not been considered by many authors, apart from [5]. This is also the case for the exterior problem (1.1)-(1.3), where as far as we know, we can only mention the work of two of the authors have done in [15]. On the other hand, in bounded domains, one can refer for instance to [6, 11, 12, 13]. Still in the case of bounded domains, we can also mentioned some numerical studies that go through the resolution the Hodge-Laplacian problem (see, e.g., $[1,11]$ ).

The paper is organized as follows. In Section 2, we introduce the Notation, the functional framework based on weighted Hilbert spaces. Section 3 is devoted to the study of problems (1.6) and (1.7), where we include the non homogeneous case for the boundary conditions. Finally, in Section 4, we consider the exterior Stokes problem (1.1)-(1.2). We first start by recall the existence and the uniqueness result of weak solution proved in [5], then we use the results established in the Section 3 to prove the existence and uniqueness of strong solutions. We finally end with the study of very weak solutions. We have deferred in the appendices the proofs of some technical results regarding the density statements or characterization of dual spaces needed to establish results on the very weak solutions.

\section{Notation and preliminaries}

\subsection{Notation}

Throughout this paper we assume that $\Omega^{\prime} \subset \mathbb{R}^{3}$ is a simply connected bounded domain that has a boundary $\Gamma$ of class $\mathscr{C}^{2,1}$. Let $\Omega$ be the complement of $\overline{\Omega^{\prime}}$ in $\mathbb{R}^{3}$, in other words an exterior domain. We assume that the origin of the coordinates is placed in the obstacle $\Omega^{\prime}$. We will use bold characters for vector and matrix fields. A point in $\mathbb{R}^{3}$ is denoted by $\boldsymbol{x}=\left(x_{1}, x_{2}, x_{3}\right)$ and its distance to the origin by

$$
r=|\boldsymbol{x}|=\left(x_{1}^{2}+x_{2}^{2}+x_{3}^{2}\right)^{1 / 2}
$$

Let $\mathbb{N}$ denote the set of non-negative integers and $\mathbb{Z}$ the set of all integers. For any multi-index $\boldsymbol{\lambda} \in \mathbb{N}^{3}$, we denote by $\partial^{\lambda}$ the differential operator of order $\lambda$,

$$
\partial^{\lambda}=\frac{\partial^{|\lambda|}}{\partial_{1}^{\lambda_{1}} \partial_{2}^{\lambda_{2}} \partial_{3}^{\lambda_{3}}}, \quad|\boldsymbol{\lambda}|=\lambda_{1}+\lambda_{2}+\lambda_{3} .
$$

For any $\alpha \in \mathbb{Z}, \mathscr{P}_{\alpha}$ stands for the space of polynomials of degree less than or equal to $\alpha$ and $\mathscr{P}_{\alpha}^{\Delta}$ the harmonic polynomials of $\mathscr{P}_{\alpha}$. If $\alpha$ is a negative integer, we set by convention $\mathscr{P}_{\alpha}=\{0\}$. We denote by $\mathscr{D}(\Omega)$ the space of $\mathscr{C}^{\infty}$ functions with compact support in $\Omega, \mathscr{D}(\bar{\Omega})$ the restriction to $\Omega$ of functions belonging to $\mathscr{D}\left(\mathbb{R}^{3}\right)$. We recall that $\mathscr{D}^{\prime}(\Omega)$ is the space of distributions defined on $\Omega$. For $m \geqslant 1$, we recall that $H^{m}(\Omega)$ is the well-known Hilbert space $W^{m, 2}(\Omega)$. We shall write $u \in H_{l o c}^{m}(\Omega)$ to mean that $u \in H^{m}(\mathscr{O})$, for any bounded domain $\mathscr{O}$, with $\overline{\mathscr{O}} \subset \Omega$. For $R>0$, we denote by $B_{R}$ the open ball of radius $R$ centered at the origin. We 
set $\Omega_{R}=\Omega \cap B_{R}$. For any $m \in \mathbb{N} \backslash\{0\}$, the space $H^{m-1 / 2}(\Gamma)$ denotes the usual trace space on $\Gamma$, of functions belonging to $H^{m}\left(\Omega_{R}\right)$ and the space $H^{-m+1 / 2}(\Gamma)$ is its dual space. The notation $\langle\cdot, \cdot\rangle$ will denote adequate duality pairing and will be specified when needed. We denote by $\langle\cdot, \cdot\rangle_{\Gamma}$ the duality pairing between the space $H^{-1 / 2}(\Gamma)$ and its dual space $H^{1 / 2}(\Gamma)$. Given a space $B$ with dual space $B^{\prime}$ and a closed subspace $X$ of $B$, we denote by $B^{\prime} \perp X$ the subspace of $B^{\prime}$ orthogonal to $X$, that is

$$
B^{\prime} \perp X=\left\{f \in B^{\prime}, \forall v \in X,\langle f, v\rangle=0\right\}=(B / X)^{\prime} .
$$

Finally, as usual, $C>0$ denotes a generic constant the value of which may change from line to line and even at the same line.

\subsection{Weighted Hilbert spaces}

We introduce the weight function

$$
\rho(\boldsymbol{x})=\left(1+r^{2}\right)^{1 / 2}
$$

For $\alpha \in \mathbb{Z}$, we introduce

$$
W_{\alpha}^{0}(\Omega)=\left\{u \in \mathscr{D}^{\prime}(\Omega), \rho^{\alpha} u \in L^{2}(\Omega)\right\},
$$

which is a Hilbert space equipped with the norm:

$$
\|u\|_{W_{\alpha}^{0}(\Omega)}=\left\|\rho^{\alpha} u\right\|_{L^{2}(\Omega)} .
$$

Let $m \geqslant 1$ be an integer. We define the weighted Hilbert space:

$$
W_{\alpha}^{m}(\Omega)=\left\{u \in \mathscr{D}^{\prime}(\Omega) ; \forall \boldsymbol{\lambda} \in \mathbb{N}^{3}: 0 \leqslant|\boldsymbol{\lambda}| \leqslant m, \rho^{\alpha-m+|\boldsymbol{\lambda}|} \partial^{\lambda} u \in L^{2}(\Omega)\right\},
$$

equipped with the norm

$$
\|u\|_{W_{\alpha}^{m}(\Omega)}=\left(\sum_{0 \leqslant|\boldsymbol{\lambda}| \leqslant m}\left\|\rho^{\alpha-m+|\boldsymbol{\lambda}|} \partial^{\lambda} u\right\|_{L^{2}(\Omega)}^{2}\right)^{1 / 2} .
$$

We define the semi-norm

$$
|u|_{W_{\alpha}^{m}(\Omega)}=\left(\sum_{|\lambda|=m}\left\|\rho^{\alpha} \partial^{\lambda} u\right\|_{L^{2}(\Omega)}\right)^{1 / 2} .
$$

Let us give examples of such space that will be often used in the remaining of the paper. For $m=1$, we have

$$
W_{\alpha}^{1}(\Omega)=\left\{u \in \mathscr{D}^{\prime}(\Omega), \rho^{\alpha-1} u \in L^{2}(\Omega), \rho^{\alpha} \nabla u \in L^{2}(\Omega)\right\}
$$

and for $m=2$,

$$
W_{\alpha+1}^{2}(\Omega)=\left\{u \in W_{\alpha}^{1}(\Omega), \rho^{\alpha+1} \partial^{2} u \in L^{2}(\Omega)\right\} .
$$

For the sake of simplicity, we have defined these spaces with integer exponents on the weight function. But 
naturally, these definitions can be extended to real number exponents with eventually some slight modifications (see[3] for more details). Observe that the space $\mathscr{D}(\bar{\Omega})$ is dense in $W_{\alpha}^{m}(\Omega)$ (see [18] or [20]). All the local properties of the space $W_{\alpha}^{m}(\Omega)$ coincide with those of the standard Hilbert space $H^{m}(\Omega)$. Hence it also satisfies the usual trace theorems on the boundary $\Gamma$. The closure of $\mathscr{D}(\Omega)$ in $W_{\alpha}^{m}(\Omega)$ is denoted by $W_{\alpha}^{m}(\Omega)$ and, as in bounded domains, can be characterized by

$$
\stackrel{\circ}{W}_{\alpha}^{m}(\Omega)=\left\{u \in W_{\alpha}^{m}(\Omega), \gamma_{0} u=0, \gamma_{1} u=0, \cdots, \gamma_{m-1} u=0 \text { on } \Gamma\right\}
$$

The dual space of $\stackrel{\circ}{\alpha}_{\alpha}^{m}(\Omega)$ is denoted by $W_{-\alpha}^{-m}(\Omega)$ equipped with the usual dual norm. This allows to extend the definition of the space $W_{\alpha}^{m}(\Omega)$ for any $\alpha$ and $m$ belonging to $\mathbb{Z}$.

We shall now give some basic properties of those spaces:

\section{Proposition 2.1.}

1. For any $\alpha, m \in \mathbb{Z}$ and for any $\boldsymbol{\lambda} \in \mathbb{N}^{3}$, the mapping

$$
u \in W_{\alpha}^{m}(\Omega) \rightarrow \partial^{\lambda} u \in W_{\alpha}^{m-|\lambda|}(\Omega)
$$

is continuous.

2. For any $\alpha, m \in \mathbb{Z}$, we have the following continuous embedding:

$$
W_{\alpha}^{m}(\Omega) \hookrightarrow W_{\alpha-1}^{m-1}(\Omega) .
$$

3. For any $\alpha, m \in \mathbb{Z}$, the space $\mathscr{P}_{m-\alpha-2}$ is the space of all polynomials included in $W_{\alpha}^{m}(\Omega)$ and if $m \geqslant 1$, the following Poincaré-type inequality holds:

$$
\forall u \in W_{\alpha}^{m}(\Omega), \quad \inf _{\lambda \in \mathscr{P}_{j^{\prime}}}\|u+\lambda\|_{W_{\alpha}^{m}(\Omega)} \leqslant C|u|_{W_{\alpha}^{m}(\Omega)}
$$

where $j^{\prime}=\min (m-\alpha-2, m-1)$. In other words the semi-norm $|\cdot|_{W_{\alpha}^{m}(\Omega)}$ is a norm on $W_{\alpha}^{m}(\Omega) / \mathscr{P}_{j^{\prime}}$. In particular, $|\cdot|_{W_{0}^{1}(\Omega)}$ is a norm on $W_{0}^{1}(\Omega)$.

4. For any $\alpha \in \mathbb{Z}, m \in \mathbb{N} \backslash\{0\}$, we have the following Poincaré-type inequality:

$$
\forall u \in \stackrel{\circ}{W}_{\alpha}^{m}(\Omega), \quad\|u\|_{W_{\alpha}^{m}(\Omega)} \leqslant C|u|_{W_{\alpha}^{m}(\Omega)} .
$$

For more details on the above properties, the reader can refer to [3, 4, 18, 20, 22] and references therein.

The result that we state below was proved by Giroire [18] and extended in weighted $L^{p}$-spaces in [3]: 
Proposition 2.2. For $m \in \mathbb{Z}$ and $\alpha \in \mathbb{Z}$, the Laplace operator defined by

$$
\Delta: W_{\alpha+m}^{m+1}\left(\mathbb{R}^{3}\right) / \mathscr{P}_{-\alpha-1}^{\Delta} \mapsto W_{\alpha+m}^{m-1}\left(\mathbb{R}^{3}\right) \perp \mathscr{P}_{\alpha-1}^{\Delta}
$$

is an isomorphism.

Remark 2.3. The isomorphism result stated in Proposition 2.2 is the property of the Laplace operator mentioned in the introduction, and that will be used to solve problem (1.7). As we also already mentioned, the main issue is to deal with the compatibility condition that appears in the right hand side of (2.12) when $\alpha \geqslant 1$.

We end this section by introducing the spaces that will be used to study problems (1.7) and (1.1) - (1.2). We first recall that for any vector field $\boldsymbol{v}=\left(v_{1}, v_{2}, v_{3}\right)$, the curl of $\boldsymbol{v}$ is defined by

$$
\operatorname{curl} \boldsymbol{v}=\left(\frac{\partial v_{3}}{\partial x_{2}}-\frac{\partial v_{2}}{\partial x_{3}}, \frac{\partial v_{1}}{\partial x_{3}}-\frac{\partial v_{3}}{\partial x_{1}}, \frac{\partial v_{2}}{\partial x_{1}}-\frac{\partial v_{1}}{\partial x_{2}}\right)
$$

Next, note that the vector-valued Laplace operator of a vector field $\boldsymbol{v}$ is equivalently defined by

$$
\Delta \boldsymbol{v}=\nabla \operatorname{div} \boldsymbol{v}-\operatorname{curl} \operatorname{curl} \boldsymbol{v}
$$

This leads to the following definitions. For $\alpha \in \mathbb{Z}$, we define

$$
\begin{gathered}
H_{\alpha}(\operatorname{curl}, \Omega)=\left\{\boldsymbol{v} \in W_{\alpha}^{0}(\Omega) ; \operatorname{curl} \boldsymbol{v} \in W_{\alpha+1}^{0}(\Omega)\right\} \\
H_{\alpha}(\operatorname{div}, \Omega)=\left\{\boldsymbol{v} \in W_{\alpha}^{0}(\Omega) ; \operatorname{div} \boldsymbol{v} \in W_{\alpha+1}^{0}(\Omega)\right\}
\end{gathered}
$$

and we set

$$
X_{\alpha}(\Omega)=H_{\alpha}(\operatorname{curl}, \Omega) \cap H_{\alpha}(\operatorname{div}, \Omega) .
$$

These spaces are respectively endowed with the norms

$$
\begin{gathered}
\|\boldsymbol{v}\|_{H_{\alpha}(\operatorname{curl}, \Omega)}=\left(\|\boldsymbol{v}\|_{W_{\alpha}^{0}(\Omega)}^{2}+\|\operatorname{curl} \boldsymbol{v}\|_{W_{\alpha+1}^{0}(\Omega)}^{2}\right)^{1 / 2}, \\
\|\boldsymbol{v}\|_{H_{\alpha}(\operatorname{div}, \Omega)}=\left(\|\boldsymbol{v}\|_{W_{\alpha}^{0}(\Omega)}^{2}+\|\operatorname{div} \boldsymbol{v}\|_{W_{\alpha+1}^{0}(\Omega)}^{2}\right)^{1 / 2}
\end{gathered}
$$

and

$$
\|\boldsymbol{v}\|_{X_{\alpha}(\Omega)}=\left(\|\boldsymbol{v}\|_{W_{\alpha}^{0}(\Omega)}^{2}+\|\operatorname{div} \boldsymbol{v}\|_{W_{\alpha+1}^{0}(\Omega)}^{2}+\|\operatorname{curl} \boldsymbol{v}\|_{W_{\alpha+1}^{0}(\Omega)}^{2}\right)^{1 / 2} .
$$

Observe that $\mathscr{D}(\bar{\Omega})$ is dense in $H_{\alpha}(\operatorname{div}, \Omega)$ and in $H_{\alpha}(\operatorname{curl}, \Omega)$. For the proof, one can use the same arguments than for the proof of the density of $\mathscr{D}(\bar{\Omega})$ in $W_{\alpha}^{m}(\Omega)$ (see $[18,20]$ ). Therefore, recalling that $\boldsymbol{n}$ is the unit normal vector to the boundary $\Gamma$ pointing outside $\Omega$, if $\boldsymbol{v}$ belongs to $H_{\alpha}(\operatorname{div}, \Omega)$, then $\boldsymbol{v}$ has normal trace $\boldsymbol{v} \cdot \boldsymbol{n}$ 
in $H^{-1 / 2}(\Gamma)$. By the same way, if $\boldsymbol{v}$ belongs to $H_{\alpha}(\mathbf{c u r l}, \Omega)$, then $\boldsymbol{v}$ has a tangential trace $\boldsymbol{v} \times \boldsymbol{n}$ that belongs to $H^{-1 / 2}(\Gamma)$. Similarly in bounded domain, we have the trace theorems: for any $\alpha \in \mathbb{Z}$, there exists $C>0$ such that

$$
\begin{gathered}
\forall \boldsymbol{v} \in H_{\alpha}(\operatorname{div}, \Omega),\|\boldsymbol{v} \cdot \boldsymbol{n}\|_{H^{-1 / 2}(\Gamma)} \leqslant C\|\boldsymbol{v}\|_{H_{\alpha}(\operatorname{div}, \Omega)}, \\
\forall \boldsymbol{v} \in H_{\alpha}(\operatorname{curl}, \Omega),\|\boldsymbol{v} \times \boldsymbol{n}\|_{H^{-1 / 2}(\Gamma)} \leqslant C\|\boldsymbol{v}\|_{H_{\alpha}(\operatorname{curl}, \Omega)} .
\end{gathered}
$$

Moreover the following Green's formulas hold. For any $\boldsymbol{v} \in H_{\alpha}(\operatorname{div}, \Omega)$ and $\varphi \in W_{-\alpha}^{1}(\Omega)$, we have

$$
\langle\boldsymbol{v} \cdot \boldsymbol{n}, \varphi\rangle_{\Gamma}=\int_{\Omega} \boldsymbol{v} \cdot \nabla \varphi \mathrm{d} \boldsymbol{x}+\int_{\Omega} \varphi \operatorname{div} \boldsymbol{v} \mathrm{d} \boldsymbol{x}
$$

and for any $\boldsymbol{v} \in H_{\alpha}($ curl, $\Omega)$ and $\boldsymbol{\varphi} \in W_{-\alpha}^{1}(\Omega)$, we have

$$
\langle\boldsymbol{v} \times \boldsymbol{n}, \boldsymbol{\varphi}\rangle_{\Gamma}=\int_{\Omega} \boldsymbol{v} \cdot \operatorname{curl} \varphi \mathrm{d} \boldsymbol{x}-\int_{\Omega} \operatorname{curl} \boldsymbol{v} \cdot \boldsymbol{\varphi} \mathrm{d} \boldsymbol{x} .
$$

We denote by $\stackrel{\circ}{H}_{\alpha}(\operatorname{div}, \Omega)$, the closure of $\mathscr{D}(\Omega)$ in $H_{\alpha}(\operatorname{div}, \Omega)$ that can be characterized as follow:

$$
\stackrel{\circ}{H}_{\alpha}(\operatorname{div}, \Omega)=\left\{\boldsymbol{v} \in H_{\alpha}(\operatorname{div}, \Omega) ; \boldsymbol{v} \cdot \boldsymbol{n}=0 \text { on } \Gamma\right\} .
$$

The dual space of $\stackrel{\circ}{H}_{\alpha}(\operatorname{div}, \Omega)$ is denoted by $H_{-\alpha}^{-1}(\operatorname{div}, \Omega)$ and is characterized by the below proposition (see [5, Proposition 2.3]).

Proposition 2.4. Assume that $\alpha \in \mathbb{Z}$. A distribution $\boldsymbol{f}$ belongs to $H_{\alpha}^{-1}(\operatorname{div}, \Omega)$ if and only if there exist $\boldsymbol{\psi} \in$ $W_{\alpha}^{0}(\Omega)$ and $\chi \in W_{\alpha-1}^{0}(\Omega)$, such that

$$
\boldsymbol{f}=\boldsymbol{\psi}+\nabla \chi
$$

Moreover we have

$$
\|\boldsymbol{\psi}\|_{W_{\alpha}^{0}(\Omega)}+\|\chi\|_{W_{\alpha-1}^{0}(\Omega)} \leqslant C\|\boldsymbol{f}\|_{H_{\alpha}^{-1}(\operatorname{div}, \Omega)} .
$$

In the sequel, we will also need the following two subspaces of $X_{\alpha}(\Omega)$ :

$$
X_{\alpha, T}(\Omega)=\left\{\boldsymbol{v} \in X_{\alpha}(\Omega) ; \boldsymbol{v} \cdot \boldsymbol{n}=0 \text { on } \Gamma\right\}
$$

and

$$
V_{\alpha, T}(\Omega)=\left\{\boldsymbol{v} \in X_{\alpha, T} ; \operatorname{div} \boldsymbol{v}=0 \text { in } \Omega\right\} .
$$

Finally, to give a sense on the second boundary condition (1.2), we introduce:

$$
G(\Delta, \Omega)=\left\{\boldsymbol{v} \in W_{0}^{1}(\Omega) ; \Delta \boldsymbol{v} \in H_{1}^{-1}(\operatorname{div}, \Omega)\right\}
$$


This is a Banach space for the norm

$$
\|\boldsymbol{v}\|_{G(\Delta, \Omega)}=\|\boldsymbol{v}\|_{W_{0}^{1}(\Omega)}+\|\Delta \boldsymbol{v}\|_{H_{1}^{-1}(\operatorname{div}, \Omega)} .
$$

Then we have the following properties (see [5, Lemma 5.1 and Corollary 5.2])

\section{Lemma 2.5 .}

i) The space $\mathscr{D}(\bar{\Omega})$ is dense in $G(\Delta, \Omega)$.

ii) The linear mapping $\gamma:\left.\boldsymbol{v} \rightarrow \operatorname{curl} \boldsymbol{\nu}\right|_{\Gamma} \times \boldsymbol{n}$ defined on $\mathscr{D}(\bar{\Omega})$ can be extended to a linear continuous mapping

$$
\gamma: G(\Delta, \Omega) \longrightarrow H^{-1 / 2}(\Gamma)
$$

Moreover, we have the Green formula: for any $\boldsymbol{v} \in G(\Delta, \Omega)$ and any $\boldsymbol{\varphi} \in V_{-1, T}(\Omega)$,

$$
-\langle\Delta \boldsymbol{v}, \boldsymbol{\varphi}\rangle_{H_{1}^{-1}(\operatorname{div}, \Omega) \times \stackrel{\circ}{H}_{-1}(\operatorname{div}, \Omega)}=\int_{\Omega} \operatorname{curl} \boldsymbol{v} \cdot \operatorname{curl} \boldsymbol{\varphi} \mathrm{d} \boldsymbol{x}-\langle\operatorname{curl} \boldsymbol{v} \times \boldsymbol{n}, \boldsymbol{\varphi}\rangle_{\Gamma} .
$$

\section{Auxiliary problems}

In this section, we deal with the auxiliary problems that are the generalized Neumann and the HodgeLaplacian problems.

\subsection{Generalized Neumann problem}

We consider here the following problem: given $\boldsymbol{f}$ and $g$, we look for a function $\pi$ satisfying

$$
\operatorname{div}(\nabla \pi-\boldsymbol{f})=0 \quad \text { in } \quad \Omega \quad \text { and } \quad(\nabla \pi-\boldsymbol{f}) \cdot \boldsymbol{n}=g \quad \text { on } \quad \Gamma .
$$

We start by looking for a solution $\pi \in W_{0}^{1}(\Omega)$ which is equivalent to look for the variational solution. Here, it is clear that (3.19) is equivalent to:

$$
\left\{\begin{array}{l}
\text { Find } \pi \in W_{0}^{1}(\Omega) \text { such that: } \\
\int_{\Omega} \nabla \pi \cdot \nabla \varphi \mathrm{d} \boldsymbol{x}=\int_{\Omega} \boldsymbol{f} \cdot \nabla \varphi \mathrm{d} \boldsymbol{x}+\langle g, \varphi\rangle_{\Gamma} .
\end{array}\right.
$$

Thanks to the Poincaré-type inequality (2.10), a straightforward application of the Lax-Milgram theorem allows to establish the following result.

Proposition 3.1. Given $(f, g) \in L^{2}(\Omega) \times H^{-1 / 2}(\Gamma)$, then (3.19) has a unique solution $\pi \in W_{0}^{1}(\Omega)$.

We continue by looking for a solution $\pi \in W_{\alpha+1}^{1}(\Omega)$ for $\alpha \neq-1$. The case $\alpha<-1$ can be solved in a straightforward manner using the isomorphism result of the Laplace operator (2.12) in $\mathbb{R}^{3}$ and the standard exterior Neumann problem. 
Theorem 3.2. Let $\alpha<-1$ be an integer and assume $(\boldsymbol{f}, g) \in W_{\alpha+1}^{0}(\Omega) \times H^{-1 / 2}(\Gamma)$. Then problem (3.19) has at least a solution $\pi \in W_{\alpha+1}^{1}(\Omega)$.

Proof. Extend $\boldsymbol{f}$ by zero in $\Omega^{\prime}$ and denote by $\tilde{\boldsymbol{f}}$ the extended function that belongs to $W_{\alpha+1}^{0}\left(\mathbb{R}^{3}\right)$. As a result, $\operatorname{div} \widetilde{\boldsymbol{f}}$ belongs to $W_{\alpha+1}^{-1}\left(\mathbb{R}^{3}\right)$ (see (2.8)) and since $\alpha<-1$ there exists $\widetilde{\pi} \in W_{\alpha+1}^{1}\left(\mathbb{R}^{3}\right)$ satisfying (see Proposition 2.2)

$$
-\Delta \widetilde{\pi}=\operatorname{div} \widetilde{\boldsymbol{f}} \text { in } \mathbb{R}^{3} .
$$

Thus it is clear that, on $\Gamma,(\nabla \widetilde{\boldsymbol{\pi}}+\widetilde{\boldsymbol{f}}) \cdot \boldsymbol{n}$ belongs to $H^{-1 / 2}(\Gamma)$. Therefore there exists a unique $z \in W_{0}^{1}(\Omega)$ satisfying (see [18] or [17, Lemma 3.7]):

$$
\Delta z=0 \quad \text { in } \quad \Omega \text { and } \nabla z \cdot \boldsymbol{n}=(\nabla \widetilde{\boldsymbol{\pi}}+\widetilde{\boldsymbol{f}}) \cdot \boldsymbol{n}+g \text { on } \Gamma \text {. }
$$

Because $\alpha<-1$, we deduce that $z$ also belongs to $W_{\alpha+1}^{1}(\Omega)$. Now setting $\pi=z-\left.\tilde{\pi}\right|_{\Omega}$, then $\pi \in W_{\alpha+1}^{1}(\Omega)$ and satisfies (3.19).

Finally, let us solve problem (3.19) when $\alpha>-1$ and to that end, we first introduce the space

$$
\mathscr{N}_{\alpha}^{\Delta}(\Omega)=\left\{\zeta \in W_{\alpha}^{1}(\Omega) ; \Delta \zeta=0 \text { in } \Omega \text { and } \nabla \zeta \cdot \boldsymbol{n}=0 \text { on } \Gamma\right\}
$$

that is the null space of the exterior Neumann problem for the Laplace operator. The below characterization has been established by Giroire [18].

Proposition 3.3. Assume $\alpha \in \mathbb{Z}$. Then we have

$$
\mathscr{N}_{\alpha}^{\Delta}(\Omega)=\left\{w(\lambda)-\lambda, \lambda \in \mathscr{P}_{-1-\alpha}^{\Delta}\right\}
$$

where $w(\lambda) \in W_{0}^{1}(\Omega)$ is the unique solution of the Neumann problem

$$
\Delta w(\lambda)=0 \quad \text { in } \quad \Omega \text { and } \nabla w(\lambda) \cdot \boldsymbol{n}=\nabla \lambda \cdot \boldsymbol{n} \text { on } \Gamma .
$$

In particular $\mathscr{N}_{\alpha}^{\Delta}(\Omega)=\{0\}$ when $\alpha \geqslant 0$ and $\mathscr{N}_{-1}^{\Delta}(\Omega)=\mathbb{R}$

Remark 3.4. Observe that, since we are dealing with an exterior domain $\Omega$ that has its boundary of class $\mathscr{C}^{2,1}$, then regularity results on the exterior Neumann problem for Laplace show that if $\zeta \in \mathscr{N}_{\alpha}^{\Delta}(\Omega)$, then $\zeta$ also belongs to $W_{\alpha+2}^{3}(\Omega)$ (see [18] or [17, theorems 3.9 and 3.10]).

Theorem 3.5. Let $\alpha>-1$ be an integer and assume $(f, g) \in W_{\alpha+1}^{0}(\Omega) \times H^{-1 / 2}(\Gamma)$ satisfies the necessary compatibility condition

$$
\forall \varphi \in \mathscr{N}_{-\alpha-1}^{\Delta}(\Omega), \quad-\int_{\Omega} \boldsymbol{f} \cdot \nabla \varphi \mathrm{d} \boldsymbol{x}=\langle g, \varphi\rangle_{\Gamma}
$$


Then problem (3.19) has at least a solution $\pi$ that belongs to $W_{\alpha+1}^{1}(\Omega)$.

Proof.

- Compatibility condition. Let us first prove that (3.22) is indeed a necessary condition. Consider $\pi \in W_{\alpha+1}^{1}(\Omega)$ a solution of (3.19) and $\varphi \in \mathscr{N}_{-\alpha-1}^{\Delta}(\Omega)$. On the one hand, using (2.13), we have

$$
\begin{aligned}
\langle g, \varphi\rangle_{\Gamma} & =\langle(\nabla \pi-\boldsymbol{f}) \cdot \boldsymbol{n}, \varphi\rangle_{\Gamma}=\int_{\Omega}(\nabla \pi-\boldsymbol{f}) \cdot \nabla \varphi \mathrm{d} \boldsymbol{x} \\
& =\int_{\Omega} \nabla \pi \cdot \nabla \varphi \mathrm{d} \boldsymbol{x}-\int_{\Omega} \boldsymbol{f} \cdot \nabla \varphi \mathrm{d} \boldsymbol{x} .
\end{aligned}
$$

On the other hand, using again (2.13) and the fact that $\varphi \in \mathscr{N}_{-\alpha-1}^{\Delta}(\Omega)$, we can write

$$
0=\langle\nabla \varphi \cdot \boldsymbol{n}, \pi\rangle_{\Gamma}=\int_{\Omega} \nabla \pi \cdot \nabla \varphi \mathrm{d} \boldsymbol{x}
$$

Summarizing, we get (3.22).

- ExistenCE. Let us now prove the existence of a solution to (3.19). Because $\alpha>-1$ and $\boldsymbol{f} \in W_{\alpha+1}^{0}(\Omega)$, then $\boldsymbol{f}$ also belongs to $L^{2}(\Omega)$. Hence Proposition 3.1 insures the existence of $\pi \in W_{0}^{1}(\Omega)$ satisfying problem (3.19). It remains now to prove that $\pi$ actually belongs to $W_{\alpha+1}^{1}(\Omega)$ and this is done using Proposition 2.2. To that end, let $\pi^{\prime} \in H^{1}\left(\Omega^{\prime}\right)$ be the unique solution of

$$
\Delta \pi^{\prime}=0 \text { in } \Omega^{\prime} \text { and } \pi^{\prime}=\pi \text { on } \Gamma \text {. }
$$

Setting now

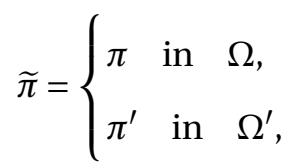

then $\widetilde{\pi}$ belongs to $W_{0}^{1}\left(\mathbb{R}^{3}\right)$. In order to use (2.12), we need to prove that $\Delta \widetilde{\pi}$ belongs to $W_{\alpha+1}^{-1}\left(\mathbb{R}^{3}\right) \perp \mathscr{P}{ }_{\alpha}^{\Delta}$. For any $\varphi \in \mathscr{D}\left(\mathbb{R}^{3}\right)$, we have

$$
\langle\Delta \tilde{\pi}, \varphi\rangle_{\mathscr{D}^{\prime}\left(\mathbb{R}^{3}\right) \times \mathscr{D}\left(\mathbb{R}^{3}\right)}=\int_{\mathbb{R}^{3}} \tilde{\pi} \Delta \varphi \mathrm{d} \boldsymbol{x}=\int_{\Omega} \pi \Delta \varphi \mathrm{d} \boldsymbol{x}+\int_{\Omega^{\prime}} \pi^{\prime} \Delta \varphi \mathrm{d} \boldsymbol{x} .
$$

Observe that

$$
\begin{aligned}
\int_{\Omega} \pi \Delta \varphi \mathrm{d} \boldsymbol{x} & =\langle\nabla \varphi \cdot \boldsymbol{n}, \pi\rangle_{\Gamma}-\int_{\Omega} \nabla \varphi \cdot \nabla \pi \mathrm{d} \boldsymbol{x} \\
& =\langle\nabla \varphi \cdot \boldsymbol{n}, \pi\rangle_{\Gamma}-\int_{\Omega}(\nabla \pi-\boldsymbol{f}) \cdot \nabla \varphi \mathrm{d} \boldsymbol{x}-\int_{\Omega} \boldsymbol{f} \cdot \nabla \varphi \mathrm{d} \boldsymbol{x} .
\end{aligned}
$$

Besides

$$
\langle g, \varphi\rangle_{\Gamma}=\langle(\nabla \pi-\boldsymbol{f}) \cdot \boldsymbol{n}, \varphi\rangle_{\Gamma}=\int_{\Omega}(\nabla \pi-\boldsymbol{f}) \cdot \nabla \varphi \mathrm{d} \boldsymbol{x} .
$$


Combining (3.26) and (3.27), we obtain

$$
\int_{\Omega} \pi \Delta \varphi \mathrm{d} \boldsymbol{x}=\langle\nabla \varphi \cdot \boldsymbol{n}, \pi\rangle_{\Gamma}-\langle g, \varphi\rangle_{\Gamma}-\int_{\Omega} \boldsymbol{f} \cdot \nabla \varphi \mathrm{d} \boldsymbol{x} .
$$

Now, thanks again to (2.13) and (3.23), we have

$$
\int_{\Omega^{\prime}} \pi^{\prime} \Delta \varphi \mathrm{d} \boldsymbol{x}=-\langle\nabla \varphi \cdot \boldsymbol{n}, \pi\rangle_{\Gamma}+\left\langle\nabla \pi^{\prime} \cdot \boldsymbol{n}, \varphi\right\rangle_{\Gamma}
$$

Adding (3.28) and (3.29), we arrive at

$$
\langle\Delta \tilde{\pi}, \varphi\rangle_{\mathscr{D}^{\prime}\left(\mathbb{R}^{3}\right) \times \mathscr{D}\left(\mathbb{R}^{3}\right)}=\left\langle\nabla \pi^{\prime} \cdot \boldsymbol{n}, \varphi\right\rangle_{\Gamma}-\langle g, \varphi\rangle_{\Gamma}-\int_{\Omega} \boldsymbol{f} \cdot \nabla \varphi \mathrm{d} \boldsymbol{x}
$$

From here, because $\boldsymbol{f} \in W_{\alpha+1}^{0}(\Omega)$, then for any $\varphi \in \mathscr{D}\left(\mathbb{R}^{3}\right)$, we have the estimate

$$
\left|\langle\Delta \tilde{\pi}, \varphi\rangle_{\mathscr{D}^{\prime}\left(\mathbb{R}^{3}\right) \times \mathscr{D}\left(\mathbb{R}^{3}\right)}\right| \leqslant C\|\varphi\|_{W_{-\alpha-1}^{1}\left(\mathbb{R}^{3}\right)}
$$

which proves that $\Delta \tilde{\pi}$ belongs to $W_{\alpha+1}^{-1}\left(\mathbb{R}^{3}\right)$.

Consider now $\lambda \in \mathscr{P}_{\alpha}^{\Delta}$ and let us prove that $\langle\Delta \tilde{\pi}, \lambda\rangle_{W_{\alpha+1}^{-1}\left(\mathbb{R}^{3}\right) \times W_{-\alpha-1}^{1}\left(\mathbb{R}^{3}\right)}=0$, which according to (3.30), amounts to prove that

$$
\int_{\Omega} \boldsymbol{f} \cdot \nabla \lambda \mathrm{d} \boldsymbol{x}+\langle g, \lambda\rangle_{\Gamma}-\left\langle\nabla \pi^{\prime} \cdot \boldsymbol{n}, \lambda\right\rangle_{\Gamma}=0
$$

Let $w(\lambda) \in W_{0}^{1}(\Omega)$ be the unique solution of (3.21). Thanks to proposition 3.3, $w(\lambda)-\lambda$ belongs to $\mathscr{N}_{-\alpha-1}^{\Delta}(\Omega)$. Besides, because $\alpha>-1, w(\lambda)$ also belongs to $W_{-\alpha-1}^{1}(\Omega)$. Then taking the first two terms of (3.31), we can write

$$
\int_{\Omega} \boldsymbol{f} \cdot \nabla \lambda \mathrm{d} \boldsymbol{x}+\langle g, \lambda\rangle_{\Gamma}=\int_{\Omega} \boldsymbol{f} \cdot \nabla w(\lambda) \mathrm{d} \boldsymbol{x}+\langle g, w(\lambda)\rangle_{\Gamma}-\int_{\Omega} \boldsymbol{f} \cdot \nabla(w(\lambda)-\lambda) \mathrm{d} \boldsymbol{x}-\langle g, w(\lambda)-\lambda\rangle_{\Gamma}
$$

But because $w(\lambda)-\lambda$ belongs to $\mathscr{N}_{-\alpha-1}^{\Delta}(\Omega)$, then thanks to the compatibility condition (3.22), the last two terms of the above equation satisfy

$$
\int_{\Omega} \boldsymbol{f} \cdot \nabla(w(\lambda)-\lambda) \mathrm{d} \boldsymbol{x}+\langle g, w(\lambda)-\lambda\rangle_{\Gamma}=0
$$

Hence (3.31) is reduced to prove that

$$
\int_{\Omega} \boldsymbol{f} \cdot \nabla w(\lambda) \mathrm{d} \boldsymbol{x}+\langle g, w(\lambda)\rangle_{\Gamma}-\left\langle\nabla \pi^{\prime} \cdot \boldsymbol{n}, \lambda\right\rangle_{\Gamma}=0
$$

For the second term of (3.32), using (2.13) and the fact that $w(\lambda)$ satisfies (3.21), we can write 


$$
\langle g, w(\lambda)\rangle_{\Gamma}=\langle(\nabla \pi-\boldsymbol{f}) \cdot \boldsymbol{n}, w(\lambda)\rangle_{\Gamma}=\int_{\Omega}(\nabla \pi-\boldsymbol{f}) \cdot \nabla w(\lambda) \mathrm{d} \boldsymbol{x}=\langle\nabla w(\lambda) \cdot \boldsymbol{n}, \pi\rangle_{\Gamma}-\int_{\Omega} \boldsymbol{f} \cdot \nabla w(\lambda) \mathrm{d} \boldsymbol{x} .
$$

Next, for the third term, using again (2.13), (3.21) and also the fact that $\pi=\pi^{\prime}$ on $\Gamma$, we have

$$
\left\langle\nabla \pi^{\prime} \cdot \boldsymbol{n}, \lambda\right\rangle_{\Gamma}=\int_{\Omega^{\prime}} \nabla \pi^{\prime} \cdot \nabla \lambda \mathrm{d} \boldsymbol{x}=\left\langle\nabla \lambda \cdot \boldsymbol{n}, \pi^{\prime}\right\rangle_{\Gamma}=\langle\nabla w(\lambda) \cdot \boldsymbol{n}, \pi\rangle_{\Gamma}
$$

Then (3.32) follows from the combination of (3.33) and (3.34).

This shows that $\Delta \tilde{\pi}$ is orthogonal to polynomials of $\mathscr{P}_{\alpha}^{\Delta}$, which implies that $\Delta \tilde{\pi}$ belongs to $W_{\alpha+1}^{-1}\left(\mathbb{R}^{3}\right) \perp \mathscr{P}_{\alpha}^{\Delta}$. Thanks to Proposition 2.2, there exists $q \in W_{\alpha+1}^{1}\left(\mathbb{R}^{3}\right)$ satisfying

$$
\Delta q=\Delta \widetilde{\pi} \text { in } \mathbb{R}^{3} .
$$

It follows from $\alpha>-1$ and Proposition 2.2 that $\widetilde{\pi}-q$ is a polynomial of $W_{0}^{1}\left(\mathbb{R}^{3}\right)$. But this space does not contain non zero polynomials (see Proposition 2.1). Thus, we deduce that $\pi$ belongs to $W_{\alpha+1}^{1}(\Omega)$.

\subsection{The Hodge-Laplacian problem with Non Standard Boundary Condition}

In this section we study the following Laplace problem: given $\boldsymbol{f}, \boldsymbol{h}, \chi$ and $g$, we look for $\boldsymbol{u}$ satisfying

$$
\left\{\begin{aligned}
-\Delta \boldsymbol{u}=\boldsymbol{f} & \text { and } \operatorname{div} \boldsymbol{u}=\chi \text { in } \Omega, \\
\boldsymbol{u} \cdot \boldsymbol{n}=g & \text { and } \operatorname{curl} \boldsymbol{u} \times \boldsymbol{n}=\boldsymbol{h} \times \boldsymbol{n} \text { on } \Gamma .
\end{aligned}\right.
$$

For $\alpha \in \mathbb{Z}$, we introduce here the space

$$
Y_{\alpha, T}(\Omega)=\left\{\boldsymbol{v} \in X_{\alpha, T}(\Omega), \operatorname{div} \boldsymbol{v}=0 \text { and } \operatorname{curl} \boldsymbol{v}=0 \text { in } \Omega\right\} .
$$

Observe that this space can be characterized as follow (see [24, Proposition 4.8])

$$
Y_{\alpha, T}(\Omega)=\left\{\nabla v, v \in \mathscr{N}_{\alpha}^{\Delta}(\Omega)\right\}
$$

In particular, if $\alpha \geqslant-1$, then we have $Y_{\alpha, T}(\Omega)=\{\mathbf{0}\}$.

The result that we state below regarding the existence and the uniqueness of weak solution to problem $\left(\mathscr{E}_{T}\right)$, was established in [5] (see Proposition 5.3).

Proposition 3.6. Assume $\chi=0, \boldsymbol{f} \in W_{1}^{0}(\Omega), g \in H^{1 / 2}(\Gamma), \boldsymbol{h} \in H^{-1 / 2}(\Gamma)$, satisfying

$$
\operatorname{div} \boldsymbol{f}=0 \text {, }
$$




$$
\boldsymbol{f} \cdot \boldsymbol{n}=\operatorname{div}_{\Gamma}(\boldsymbol{h} \times \boldsymbol{n}) \quad \text { on } \quad \Gamma,
$$

and

$$
\forall \boldsymbol{\varphi} \in Y_{-1, T}(\Omega), \quad \int_{\Omega} \boldsymbol{f} \cdot \boldsymbol{\varphi} \mathrm{d} \boldsymbol{x}+\langle\boldsymbol{h} \times \boldsymbol{n}, \boldsymbol{\varphi}\rangle_{\Gamma}=0,
$$

then problem $\left(\mathscr{E}_{T}\right)$ has a unique solution $\boldsymbol{u} \in W_{0}^{1}(\Omega)$.

Our aim here is to establish the existence of strong solutions to $\left(\mathscr{E}_{T}\right)$ that belong to the space $W_{\alpha+1}^{2}(\Omega)$, for any $\alpha \in \mathbb{Z}$. We begin by stating results regarding the necessary conditions that the data of problem $\left(\mathscr{E}_{T}\right)$ satisfy .

Proposition 3.7. If $\boldsymbol{u} \in W_{\alpha+1}^{2}(\Omega)$ is a solution of $\left(\mathscr{E}_{T}\right)$, with data $\boldsymbol{f} \in W_{\alpha+1}^{0}(\Omega), \chi \in W_{\alpha+1}^{1}(\Omega), g \in H^{3 / 2}(\Gamma)$ and $\boldsymbol{h} \in H^{1 / 2}(\Gamma)$, then necessarily we have

$$
\begin{gathered}
\operatorname{div}(\boldsymbol{f}+\nabla \chi)=0 \quad \text { in } \quad \Omega, \\
(\boldsymbol{f}+\nabla \chi) \cdot \boldsymbol{n}=\operatorname{div}_{\Gamma}(\boldsymbol{h} \times \boldsymbol{n}) \quad \text { on } \quad \Gamma .
\end{gathered}
$$

and if $\alpha>0$,

$$
\forall \boldsymbol{\varphi} \in Y_{-\alpha-1, T}(\Omega), \quad \int_{\Omega} \boldsymbol{f} \cdot \boldsymbol{\varphi} \mathrm{d} \boldsymbol{x}+\langle\boldsymbol{h} \times \boldsymbol{n}, \boldsymbol{\varphi}\rangle_{\Gamma}=0 .
$$

Proof. The necessary condition (3.37) is straightforward by taking the divergence of the first equation of $\left(\mathscr{E}_{T}\right)$. Next, in order to establish the second necessary condition (3.38), let $\boldsymbol{u} \in W_{\alpha+1}^{2}(\Omega)$ be a solution of $\left(\mathscr{E}_{T}\right)$ and consider $\boldsymbol{z}=$ curl $\boldsymbol{u}$. It is clear that $\boldsymbol{z}$ belongs to $H_{\alpha}(\operatorname{div}, \Omega)$ and we can write

$$
\begin{aligned}
\forall \varphi \in \mathscr{D}(\bar{\Omega}), \quad\langle\operatorname{curl} \boldsymbol{z} \cdot \boldsymbol{n}, \varphi\rangle_{\Gamma} & =\int_{\Omega} \operatorname{curl} \boldsymbol{z} \cdot \nabla \varphi \mathrm{d} \boldsymbol{x} \\
& =-\langle\boldsymbol{z} \times \boldsymbol{n}, \nabla \varphi\rangle_{\Gamma} \\
& =\left\langle\operatorname{div}_{\Gamma}(\boldsymbol{z} \times \boldsymbol{n}), \varphi\right\rangle_{\Gamma} \\
& =\left\langle\operatorname{div}_{\Gamma}(\boldsymbol{h} \times \boldsymbol{n}), \varphi\right\rangle_{\Gamma} .
\end{aligned}
$$

As a consequence,

$$
\langle(\nabla \operatorname{div} \boldsymbol{u}-\Delta \boldsymbol{u}) \cdot \boldsymbol{n}, \varphi\rangle_{\Gamma}=\left\langle\operatorname{div}_{\Gamma}(\boldsymbol{h} \times \boldsymbol{n}), \varphi\right\rangle_{\Gamma}
$$

and

$$
\langle(\boldsymbol{f}+\nabla \chi) \cdot \boldsymbol{n}, \varphi\rangle_{\Gamma}=\left\langle\operatorname{div}_{\Gamma}(\boldsymbol{h} \times \boldsymbol{n}), \varphi\right\rangle_{\Gamma} .
$$

Then (3.38) holds in the $H^{-1 / 2}(\Gamma)$ sense due to the density of $\mathscr{D}(\bar{\Omega})$ in $W_{\alpha}^{1}(\Omega)$ for any $\alpha \in \mathbb{Z}$.

Finally, let us prove (3.39). Observe that because of the characterization of the space $Y_{-1-\alpha, T}(\Omega)$, the compatibility condition (3.39) only appears for the case $\alpha>0$. Let $\boldsymbol{u} \in W_{\alpha+1}^{2}(\Omega)$ be again a solution of $\left(\mathscr{E}_{T}\right)$. Multiplying the first equation of $\left(\mathscr{E}_{T}\right)$ by $\phi \in X_{-\alpha-1, T}(\Omega)$ and integrating, give

$$
\int_{\Omega}-\Delta \boldsymbol{u} \cdot \boldsymbol{\phi} \mathrm{d} \boldsymbol{x}=\int_{\Omega} \boldsymbol{f} \cdot \boldsymbol{\phi} \mathrm{d} \boldsymbol{x}
$$


which can be writing as

$$
\int_{\Omega} \operatorname{curl} \operatorname{curl} \boldsymbol{u} \cdot \boldsymbol{\phi} \mathrm{d} \boldsymbol{x}-\int_{\Omega} \nabla \chi \cdot \boldsymbol{\phi} \mathrm{d} \boldsymbol{x}=\int_{\Omega} \boldsymbol{f} \cdot \boldsymbol{\phi} \mathrm{d} \boldsymbol{x} .
$$

It follows from (2.13) and (2.14), that

$$
\int_{\Omega} \operatorname{curl} \boldsymbol{u} \cdot \operatorname{curl} \phi \mathrm{d} \boldsymbol{x}=\int_{\Omega} \boldsymbol{f} \cdot \boldsymbol{\phi} \mathrm{d} \boldsymbol{x}-\int_{\Omega} \chi \operatorname{div} \boldsymbol{\phi} \mathrm{d} \boldsymbol{x}+\langle\boldsymbol{h} \times \boldsymbol{n}, \boldsymbol{\phi}\rangle_{\Gamma} .
$$

As a result, we see that the data $\boldsymbol{f}, \chi$ and $\boldsymbol{h}$ satisfy the necessary condition

$$
\forall \boldsymbol{\phi} \in X_{-\alpha-1, T}(\Omega), \text { s.t. } \operatorname{curl} \boldsymbol{\phi}=\mathbf{0}, \quad \int_{\Omega} \boldsymbol{f} \cdot \boldsymbol{\phi} \mathrm{d} \boldsymbol{x}-\int_{\Omega} \chi \operatorname{div} \boldsymbol{\phi} \mathrm{d} \boldsymbol{x}+\langle\boldsymbol{h} \times \boldsymbol{n}, \boldsymbol{\phi}\rangle_{\Gamma}=0 .
$$

Then for any $\boldsymbol{\varphi} \in Y_{-\alpha-1, T}(\Omega) \subset X_{-\alpha-1, T}(\Omega)$, we clearly have (3.39). Conversely, if (3.39) holds, then (3.40) also holds. Indeed, let $\boldsymbol{\phi}$ be in $X_{-\alpha-1, T}(\Omega)$ such that curl $\boldsymbol{\phi}=\mathbf{0}$. Then, because $\alpha>0$, the problem

$$
\Delta v=\operatorname{div} \boldsymbol{\phi} \text { in } \Omega \text { and } \nabla v \cdot \boldsymbol{n}=0 \text { on } \Gamma \text {, }
$$

has a solution $v \in W_{-\alpha}^{2}(\Omega)$ (see [17, Theorem 3.9]). Set now $\boldsymbol{\varphi}=\boldsymbol{\phi}-\nabla \nu$, then obviously $\boldsymbol{\varphi} \in Y_{-\alpha-1, T}(\Omega)$ and, thanks to (3.39), we can write

$$
\int_{\Omega} \boldsymbol{f} \cdot \boldsymbol{\phi} \mathrm{d} \boldsymbol{x}-\int_{\Omega} \boldsymbol{f} \cdot \nabla v \mathrm{~d} \boldsymbol{x}+\langle\boldsymbol{h} \times \boldsymbol{n}, \boldsymbol{\phi}\rangle_{\Gamma}-\langle\boldsymbol{h} \times \boldsymbol{n}, \nabla v\rangle_{\Gamma}=0 .
$$

It follows from (3.37), (3.38) and (2.13), that

$$
\begin{aligned}
-\langle\boldsymbol{h} \times \boldsymbol{n}, \nabla v\rangle_{\Gamma} & =\left\langle\operatorname{div}_{\Gamma}(\boldsymbol{h} \times \boldsymbol{n}), v\right\rangle_{\Gamma}=\langle(\boldsymbol{f}+\nabla \chi) \cdot \boldsymbol{n}, v\rangle_{\Gamma} \\
& =\int_{\Omega}(\boldsymbol{f}+\nabla \chi) \cdot \nabla v \mathrm{~d} \boldsymbol{x}+\int_{\Omega} v \operatorname{div}(\boldsymbol{f}+\nabla \chi) \mathrm{d} \boldsymbol{x} \\
& =\int_{\Omega}(\boldsymbol{f}+\nabla \chi) \cdot \nabla v \mathrm{~d} \boldsymbol{x} .
\end{aligned}
$$

This shows that

$$
-\int_{\Omega} \boldsymbol{f} \cdot \nabla v \mathrm{~d} \boldsymbol{x}-\langle\boldsymbol{h} \times \boldsymbol{n}, \nabla \nu\rangle_{\Gamma}=\int_{\Omega} \nabla \chi \cdot \nabla \nu \mathrm{d} \boldsymbol{x} .
$$

Moreover, using (2.13) and the fact that $\nabla v \cdot \boldsymbol{n}=0$ on $\Gamma$ allow to obtain

$$
\int_{\Omega} \nabla \chi \cdot \nabla v \mathrm{~d} \boldsymbol{x}=-\int_{\Omega} \chi \operatorname{div} \boldsymbol{\phi} \mathrm{d} \boldsymbol{x}
$$

Then (3.40) follows from the combination of (3.41), (3.42) and (3.43).

Remark 3.8. In the course of the latter proof, we can observe that, on the one hand, condition (3.40) is naturally satisfied by the data, and on the other hand, we showed the equivalence between (3.39) and (3.40). The choice of using (3.39) in order to express the necessary condition that satisfy the data, is motivated by the study 
of the exterior Stokes problem (1.1)-(1.2) where it will be more convenient to verify (3.39).

Before stating results on strong solutions, let us make a further remark.

Remark 3.9. Let $R>0$ be a real number large enough so that $\overline{\Omega^{\prime}} \subset B_{R}$. We recall that $\Omega_{R}=\Omega \cap B_{R}$. In what follows, we shall use the fact that if $\boldsymbol{u} \in H^{1}\left(\Omega_{R}\right)$ solves the following mixed boundary value problem:

$$
\left\{\begin{array}{l}
-\Delta \boldsymbol{u}=\boldsymbol{f} \text { and } \operatorname{div} \boldsymbol{u}=\chi \text { in } \Omega_{R}, \\
\boldsymbol{u} \cdot \boldsymbol{n}=g \text { and } \operatorname{curl} \boldsymbol{u} \times \boldsymbol{n}=\boldsymbol{h} \times \boldsymbol{n} \text { on } \Gamma, \\
\boldsymbol{u}=\boldsymbol{a} \text { on } \partial B_{R},
\end{array}\right.
$$

with data $\boldsymbol{f} \in L^{2}\left(\Omega_{R}\right), \chi \in H^{1}\left(\Omega_{R}\right), g \in H^{3 / 2}(\Gamma), \boldsymbol{h} \in H^{1 / 2}(\Gamma)$ and $\boldsymbol{a} \in H^{3 / 2}\left(\partial B_{R}\right)$, then we have $\boldsymbol{u} \in H^{2}\left(\Omega_{R}\right)$. Indeed, because the two components $\Gamma$ and $\partial B_{R}$ of the boundary are completely disconnected, we can use a partition of unity to establish the regularity result. The proof is postponed in Section A.

We now split the statements on the existence of strong solutions into two parts, depending on the values of $\alpha$.

Theorem 3.10. Let $\alpha \leqslant 0$ be an integer. Assume $\boldsymbol{f} \in W_{\alpha+1}^{0}(\Omega), \chi \in W_{\alpha+1}^{1}(\Omega), g \in H^{3 / 2}(\Gamma) \boldsymbol{h} \in H^{1 / 2}(\Gamma)$ satisfying (3.37) and (3.38). Then problem $\left(\mathscr{E}_{T}\right)$ has at least a solution $\boldsymbol{u} \in W_{\alpha+1}^{2}(\Omega)$.

Proof. We divide the proof into two steps.

- STEP 1. This step is dedicated to the proof of the existence of $\boldsymbol{u} \in W_{\alpha}^{1}(\Omega)$ solution of $\left(\mathscr{E}_{T}\right)$.

We first consider the case $\chi=0$. Then the key point is to construct an extension of the datum $f$ in such a way that the extension is divergence free which in turn allows get a divergence free solution of the Hodge-Laplacian in the whole space $\mathbb{R}^{3}$. To that end, observe that in view of (3.38), for all $\varphi \in H^{2}\left(\Omega^{\prime}\right)$, we have

$$
\langle\boldsymbol{f} \cdot \boldsymbol{n}, \varphi\rangle_{\Gamma}=\left\langle\operatorname{div}_{\Gamma}(\boldsymbol{h} \times \boldsymbol{n}), \varphi\right\rangle_{\Gamma}=-\langle\boldsymbol{h} \times \boldsymbol{n}, \nabla \varphi\rangle_{\Gamma}
$$

In particular, if $\varphi=1$, we get:

$$
\langle\boldsymbol{f} \cdot \boldsymbol{n}, 1\rangle_{\Gamma}=0
$$

Consider now the following Neumann problem in the bounded domain $\Omega^{\prime}$

$$
\Delta \theta=0 \quad \text { in } \quad \Omega^{\prime} \text { and } \nabla \theta \cdot \boldsymbol{n}=\boldsymbol{f} \cdot \boldsymbol{n} \text { on } \Gamma .
$$


Owing to condition (3.46), problem (3.47) has a solution $\theta \in H^{1}\left(\Omega^{\prime}\right)$. Let us now set

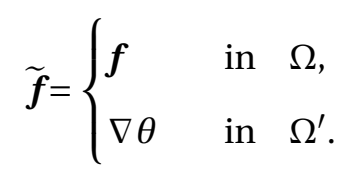

Then, on the one hand, clearly $\tilde{\boldsymbol{f}}$ belongs to $W_{\alpha+1}^{0}\left(\mathbb{R}^{3}\right)$, and because $\alpha \leqslant 0$, Proposition 2.2 yields the existence of $\widetilde{\boldsymbol{v}} \in W_{\alpha+1}^{2}\left(\mathbb{R}^{3}\right)$, satisfying

$$
-\Delta \widetilde{\boldsymbol{v}}=\widetilde{\boldsymbol{f}} \text { in } \mathbb{R}^{3}
$$

On the other hand, for any $\varphi \in \mathscr{D}\left(\mathbb{R}^{3}\right)$, we can write

$$
\begin{aligned}
\langle\operatorname{div} \widetilde{\boldsymbol{f}}, \varphi\rangle_{\mathscr{D}^{\prime}\left(\mathbb{R}^{3}\right) \times \mathscr{D}\left(\mathbb{R}^{3}\right)} & =-\int_{\mathbb{R}^{3}} \widetilde{\boldsymbol{f}} \cdot \nabla \varphi \mathrm{d} \boldsymbol{x} \\
& =-\int_{\Omega} \boldsymbol{f} \cdot \nabla \varphi \mathrm{d} \boldsymbol{x}-\int_{\Omega^{\prime}} \nabla \theta \cdot \nabla \varphi \mathrm{d} \boldsymbol{x} \\
& =-\langle\boldsymbol{f} \cdot \boldsymbol{n}, \varphi\rangle_{\Gamma}+\langle\nabla \theta \cdot \boldsymbol{n}, \varphi\rangle_{\Gamma} \\
& =0
\end{aligned}
$$

which implies that $\operatorname{div} \widetilde{\boldsymbol{f}}=0$ in $\mathbb{R}^{3}$. Therefore, taking the divergence of (3.48) and thanks again to Proposition 2.2, we deduce that $\operatorname{div} \widetilde{\boldsymbol{v}}$ is a harmonic polynomial of $W_{\alpha+1}^{1}\left(\mathbb{R}^{3}\right)$ which means that there exists $p \in \mathscr{P}_{-\alpha-2}^{\Delta}$ such that $\operatorname{div} \widetilde{\boldsymbol{v}}=p$. Using now a characterization of harmonic polynomials (see [16, Lemma 4.1]), there exists $\boldsymbol{q} \in \mathscr{P}_{-\alpha-1}^{\Delta}$ such that $p=\operatorname{div} \boldsymbol{q}$. Hence, setting $\widetilde{\boldsymbol{u}}=\widetilde{\boldsymbol{v}}-\boldsymbol{q} \in W_{\alpha+1}^{2}\left(\mathbb{R}^{3}\right)$, then we have

$$
-\Delta \widetilde{\boldsymbol{u}}=\widetilde{\boldsymbol{f}} \quad \text { in } \quad \mathbb{R}^{3} \quad \text { and } \quad \operatorname{div} \widetilde{\boldsymbol{u}}=0 \quad \text { in } \quad \mathbb{R}^{3} .
$$

Next, let us introduce the following problem

$$
\left\{\begin{array}{l}
\Delta z=\mathbf{0} \text { and } \operatorname{div} z=0 \text { in } \Omega, \\
\boldsymbol{z} \cdot \boldsymbol{n}=-\widetilde{\boldsymbol{u}} \cdot \boldsymbol{n}+g \text { and } \operatorname{curl} \boldsymbol{z} \times \boldsymbol{n}=(-\operatorname{curl} \widetilde{\boldsymbol{u}}+\boldsymbol{h}) \times \boldsymbol{n} \text { on } \Gamma
\end{array}\right.
$$

In order to apply Proposition 3.6, we need to prove (3.35) and (3.36) for the above problem. For any 
$\varphi \in \mathscr{D}(\bar{\Omega})$, we can write:

$$
\begin{aligned}
\left\langle\operatorname{div}_{\Gamma}(-\operatorname{curl} \widetilde{\boldsymbol{u}} \times \boldsymbol{n}+\boldsymbol{h} \times \boldsymbol{n}), \varphi\right\rangle_{\Gamma} & =\left\langle\operatorname{div}_{\Gamma}(-\operatorname{curl} \widetilde{\boldsymbol{u}} \times \boldsymbol{n}), \varphi\right\rangle_{\Gamma}+\left\langle\operatorname{div}_{\Gamma}(\boldsymbol{h} \times \boldsymbol{n}), \varphi\right\rangle_{\Gamma} \\
& =\langle\operatorname{curl} \widetilde{\boldsymbol{u}} \times \boldsymbol{n}, \nabla \varphi\rangle_{\Gamma}+\left\langle\operatorname{div}_{\Gamma}(\boldsymbol{h} \times \boldsymbol{n}), \varphi\right\rangle_{\Gamma} \\
& =-\int_{\Omega} \operatorname{curl} \operatorname{curl} \widetilde{\boldsymbol{u}} \cdot \nabla \varphi \mathrm{d} \boldsymbol{x}+\left\langle\operatorname{div}_{\Gamma}(\boldsymbol{h} \times \boldsymbol{n}), \varphi\right\rangle_{\Gamma} \\
& =\int_{\Omega} \Delta \tilde{\boldsymbol{u}} \cdot \nabla \varphi \mathrm{d} \boldsymbol{x}+\left\langle\operatorname{div}_{\Gamma}(\boldsymbol{h} \times \boldsymbol{n}), \varphi\right\rangle_{\Gamma} \\
& =-\int_{\Omega} \tilde{\boldsymbol{f}} \cdot \nabla \varphi \mathrm{d} \boldsymbol{x}+\left\langle\operatorname{div}_{\Gamma}(\boldsymbol{h} \times \boldsymbol{n}), \varphi\right\rangle_{\Gamma} \\
& =-\langle\boldsymbol{f} \cdot \boldsymbol{n}, \varphi\rangle_{\Gamma}+\left\langle\operatorname{div}_{\Gamma}(\boldsymbol{h} \times \boldsymbol{n}), \varphi\right\rangle_{\Gamma} \\
& =0 .
\end{aligned}
$$

Then (3.35) follows from the density of $\mathscr{D}(\bar{\Omega})$ in $W_{\beta}^{1}(\Omega)$ for any $\beta \in \mathbb{Z}$.

Next, to prove (3.36) for problem (3.49), let us take $\boldsymbol{\varphi} \in Y_{-1, T}(\Omega)$. Owing to the characterization of the space $Y_{-1, T}(\Omega)$, we have $\boldsymbol{\varphi}=\nabla w(\lambda)$, for $\lambda \in \mathbb{R}$ and $w(\lambda) \in W_{0}^{1}(\Omega)$ is the unique solution of (3.21). Then using the above relations, we can write

$$
\begin{aligned}
\langle(-\operatorname{curl} \widetilde{\boldsymbol{u}}+\boldsymbol{h}) \times \boldsymbol{n}, \boldsymbol{\varphi}\rangle_{\Gamma} & =\langle(-\operatorname{curl} \widetilde{\boldsymbol{u}}+\boldsymbol{h}) \times \boldsymbol{n}, \nabla w(\lambda)\rangle_{\Gamma} \\
& =-\left\langle\operatorname{div}_{\Gamma}((-\operatorname{curl} \widetilde{\boldsymbol{u}}+\boldsymbol{h}) \times \boldsymbol{n}), w(\lambda)\right\rangle_{\Gamma} \\
& =0 .
\end{aligned}
$$

Consequently, thanks to Proposition 3.6, problem (3.49) has a solution $z \in W_{0}^{1}(\Omega) \subset W_{\alpha}^{1}(\Omega)$ since $\alpha \leqslant 0$. Setting now $\boldsymbol{u}=\boldsymbol{z}+\left.\widetilde{\boldsymbol{u}}\right|_{\Omega}$, then $\boldsymbol{u}$ belongs to $W_{\alpha}^{1}(\Omega)$ and satisfies $\left(\mathscr{E}_{T}\right)$.

We now consider the case where $\chi \in W_{\alpha+1}^{1}(\Omega)$. Let us introduce the following problem:

$$
-\Delta v=\chi \text { in } \Omega \text { and } \nabla \nu \cdot \boldsymbol{n}=0 \text { on } \Gamma \text {. }
$$

Since $\alpha<0$, this problem has at least a solution $v \in W_{\alpha+1}^{2}(\Omega$ (see [17, Theorem 3.9]). Consider the following problem:

$$
\left\{\begin{array}{l}
-\Delta \boldsymbol{z}=\boldsymbol{f}+\nabla \chi \quad \text { and } \operatorname{div} \boldsymbol{z}=0 \text { in } \Omega, \\
\boldsymbol{z} \cdot \boldsymbol{n}=g \text { and } \operatorname{curl} \boldsymbol{z} \times \boldsymbol{n}=\boldsymbol{h} \times \boldsymbol{n} \text { on } \Gamma .
\end{array}\right.
$$

Because the data satisfy (3.37) and (3.38), then the previous case allows to establish the existence of $z \in W_{\alpha+1}^{1}(\Omega)$ solution to (3.50). We thus deduce that $\boldsymbol{u}=\boldsymbol{z}-\nabla v \in W_{\alpha}^{1}(\Omega)$ is a solution of problem $\left(\mathscr{E}_{T}\right)$.

- STEP 2. In this step, we prove that the solution $\boldsymbol{u} \in W_{\alpha}^{1}(\Omega)$ established in the first step, also belongs to $W_{\alpha+1}^{2}(\Omega)$ due to the assumptions on the data and the fact that the boundary $\Gamma$ is of class $\mathscr{C}^{2,1}$. The 
regularity result can be established by combining the Laplace problem in the whole space $\mathbb{R}^{3}$ and the mixed boundary value problem for Laplace discussed in Remark 3.9. So let $R>0$ be a real number large enough so that $\overline{\Omega^{\prime}} \subset B_{R}$ and consider the following partition of unity:

$$
\begin{aligned}
& \psi_{1}, \psi_{2} \in \mathscr{C}^{\infty}\left(\mathbb{R}^{3}\right), \quad 0 \leqslant \psi_{1}, \psi_{2} \leqslant 1, \quad \psi_{1}+\psi_{2}=1 \quad \text { in } \mathbb{R}^{3} \\
& \psi_{1}=1 \quad \text { in } B_{R}, \quad \operatorname{supp} \psi_{1} \subset B_{R+1} .
\end{aligned}
$$

Next, extend $\boldsymbol{f}$ by zero in $\Omega^{\prime}$ and denote by $\widetilde{\boldsymbol{f}} \in W_{\alpha+1}^{0}\left(\mathbb{R}^{3}\right)$ the extended function. Moreover, let $\widetilde{\boldsymbol{u}} \in$ $W_{\alpha}^{1}\left(\mathbb{R}^{3}\right)$ be an extension of $\boldsymbol{u}$. Then $\widetilde{\boldsymbol{u}}$ can be written as:

$$
\widetilde{\boldsymbol{u}}=\psi_{1} \widetilde{\boldsymbol{u}}+\psi_{2} \widetilde{\boldsymbol{u}}=\widetilde{\boldsymbol{u}}_{1}+\widetilde{\boldsymbol{u}}_{2}
$$

Next, one can easily observe that $\widetilde{\boldsymbol{u}}_{2}$ satisfies:

$$
-\Delta \widetilde{\boldsymbol{u}}_{2}=\widetilde{\boldsymbol{f}}_{2} \text { in } \mathbb{R}^{3}
$$

where $\widetilde{\boldsymbol{f}}_{2}=\psi_{2} \widetilde{\boldsymbol{f}}-\Delta \psi_{2} \widetilde{\boldsymbol{u}}-2 \nabla \psi_{2} \cdot \nabla \widetilde{\boldsymbol{u}}$. Owing to the support of $\psi_{2}, \widetilde{\boldsymbol{f}}_{2}$ has the same regularity as $\widetilde{\boldsymbol{f}}$ and so belongs to $W_{\alpha+1}^{0}\left(\mathbb{R}^{3}\right)$. It follows from Proposition 2.2 that there exists $\tilde{z} \in W_{\alpha+1}^{2}\left(\mathbb{R}^{3}\right)$ satisfying

$$
-\Delta \widetilde{\boldsymbol{z}}=\widetilde{\boldsymbol{f}}_{2} \text { in } \mathbb{R}^{3} .
$$

This implies that $\widetilde{\boldsymbol{u}}_{2}-\tilde{\boldsymbol{z}} \in W_{\alpha}^{1}\left(\mathbb{R}^{3}\right)$ is a harmonic tempered distribution and therefore a harmonic polynomial that belongs to $\mathscr{P}_{-\alpha-1}^{\Delta}$. The fact that $\mathscr{P}_{-\alpha-1}^{\Delta} \subset W_{\alpha+1}^{2}\left(\mathbb{R}^{3}\right)$ yields that $\widetilde{\boldsymbol{u}}_{2}$ also belongs to $W_{\alpha+1}^{2}\left(\mathbb{R}^{3}\right)$. In particular, we have $\widetilde{\boldsymbol{u}}_{2}=\boldsymbol{u}$ outside $B_{R+1}$, so the restriction of $\boldsymbol{u}$ to $\partial B_{R+1}$ belongs to $H^{3 / 2}(\Gamma)$. Therefore, $\boldsymbol{u}$ satisfies:

$$
\left\{\begin{array}{l}
-\Delta \boldsymbol{u}=\boldsymbol{f} \text { and } \operatorname{div} \boldsymbol{u}=\chi \text { in } \Omega_{R+1}, \\
\boldsymbol{u} \cdot \boldsymbol{n}=g \text { and } \operatorname{curl} \boldsymbol{u} \times \boldsymbol{n}=\boldsymbol{h} \times \boldsymbol{n} \text { on } \Gamma, \\
\boldsymbol{u}=\widetilde{\boldsymbol{u}}_{2} \text { on } \partial B_{R+1},
\end{array}\right.
$$

It follows from Remark 3.9 and the assumptions on the data and on the boundary $\Gamma$, that $\boldsymbol{u}$ belongs to $H^{2}\left(\Omega_{R+1}\right)$, which in turn shows that $\widetilde{\boldsymbol{u}}_{1}$ also belongs to $H^{2}\left(\Omega_{R+1}\right)$. Thus we can conclude that $\boldsymbol{u}$ also belongs to $W_{\alpha+1}^{2}(\Omega)$.

We now look for strong solutions of problem $\left(\mathscr{E}_{T}\right)$, when $\alpha>0$.

Theorem 3.11. Let $\alpha>0$ be an integer and assume $\boldsymbol{f} \in W_{\alpha+1}^{0}(\Omega), \chi \in W_{\alpha+1}^{1}(\Omega), g \in H^{3 / 2}(\Gamma)$ and $\boldsymbol{h} \in H^{1 / 2}(\Gamma)$

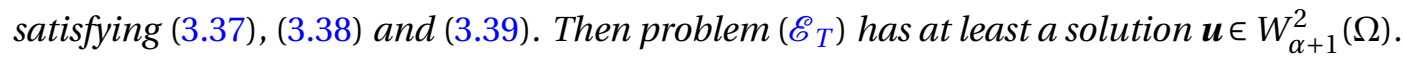


Proof. Observe that it enough to prove the existence of a solution $\boldsymbol{u}$ that belongs to $W_{\alpha}^{1}(\Omega)$. Indeed, one can then proceed as in the step 2 of the proof of Theorem 3.10 to show that $\boldsymbol{u}$ also belongs to $W_{\alpha+1}^{2}(\Omega)$. Now, to prove the existence of a solution $\boldsymbol{u}$ that belongs to $W_{\alpha}^{1}(\Omega)$, we split the proof in two cases

- THE CASE $g=0$. Because $\alpha>0$, then $\boldsymbol{f} \in L^{2}(\Omega), \chi \in W_{0}^{1}(\Omega)$ and by virtue of Theorem 3.10, problem $\left(\mathscr{E}_{T}\right)$ has a solution $\boldsymbol{u}$ in $W_{0}^{2}(\Omega)$. It remains now to prove that $\boldsymbol{u}$ belongs to $W_{\alpha}^{1}(\Omega)$. This is again based on the properties of the Laplace equation in the whole space $\mathbb{R}^{3}$. To that end, let $\boldsymbol{u}^{\prime} \in H^{2}\left(\Omega^{\prime}\right)$ be the unique solution of

$$
\Delta \boldsymbol{u}^{\prime}=0 \quad \text { in } \quad \Omega^{\prime} \quad \text { and } \quad \boldsymbol{u}^{\prime}=\boldsymbol{u} \text { on } \Gamma \text {. }
$$

Set now

$$
\widetilde{\boldsymbol{u}}=\left\{\begin{array}{lll}
\boldsymbol{u} & \text { in } & \Omega, \\
\boldsymbol{u}^{\prime} & \text { in } & \Omega^{\prime} .
\end{array}\right.
$$

It is clear that $\widetilde{\boldsymbol{u}}$ belongs to $W_{-1}^{1}\left(\mathbb{R}^{3}\right)$. Set $\boldsymbol{F}=-\Delta \widetilde{\boldsymbol{u}}$ and let us prove that $\boldsymbol{F}$ belongs to $W_{\alpha}^{-1}\left(\mathbb{R}^{3}\right)$. For any $\boldsymbol{\varphi} \in \mathscr{D}\left(\mathbb{R}^{3}\right)$, we have

$$
\begin{aligned}
\langle\boldsymbol{F}, \boldsymbol{\varphi}\rangle_{\mathscr{D}^{\prime}\left(\mathbb{R}^{3}\right) \times \mathscr{D}\left(\mathbb{R}^{3}\right)} & =-\int_{\mathbb{R}^{3}} \widetilde{\boldsymbol{u}} \cdot \Delta \boldsymbol{\varphi} \mathrm{d} \boldsymbol{x} \\
& =-\int_{\Omega} \boldsymbol{u} \cdot \Delta \boldsymbol{\varphi} \mathrm{d} \boldsymbol{x}-\int_{\Omega^{\prime}} \boldsymbol{u}^{\prime} \cdot \Delta \boldsymbol{\varphi} \mathrm{d} \boldsymbol{x} \\
& =\int_{\Omega} \boldsymbol{u} \cdot(\operatorname{curl} \operatorname{curl} \boldsymbol{\varphi}-\nabla \operatorname{div} \boldsymbol{\varphi}) \mathrm{d} \boldsymbol{x}+\int_{\Omega^{\prime}} \boldsymbol{u}^{\prime} \cdot(\operatorname{curl} \operatorname{curl} \boldsymbol{\varphi}-\nabla \operatorname{div} \boldsymbol{\varphi}) \mathrm{d} \boldsymbol{x} \\
& =\int_{\Omega} \operatorname{curl} \boldsymbol{u} \cdot \operatorname{curl} \boldsymbol{\varphi} \mathrm{d} \boldsymbol{x}+\int_{\Omega^{\prime}} \operatorname{curl} \boldsymbol{u}^{\prime} \cdot \operatorname{curl} \boldsymbol{\varphi} \mathrm{d} \boldsymbol{x}+\int_{\Omega} \chi \operatorname{div} \boldsymbol{\varphi} \mathrm{d} \boldsymbol{x}+\int_{\Omega^{\prime}} \operatorname{div} \boldsymbol{u}^{\prime} \operatorname{div} \boldsymbol{\varphi} \mathrm{d} \boldsymbol{x} \\
& =\int_{\Omega} \boldsymbol{f} \cdot \boldsymbol{\varphi} \mathrm{d} \boldsymbol{x}+\langle\boldsymbol{h} \times \boldsymbol{n}, \boldsymbol{\varphi}\rangle_{\Gamma}+\langle\boldsymbol{\varphi} \cdot \boldsymbol{n}, \chi\rangle_{\Gamma}-\left\langle\boldsymbol{\varphi} \cdot \boldsymbol{n}, \operatorname{div} \boldsymbol{u}^{\prime}\right\rangle_{\Gamma}-\left\langle\operatorname{curl} \boldsymbol{u}^{\prime} \times \boldsymbol{n}, \boldsymbol{\varphi}\right\rangle_{\Gamma} .
\end{aligned}
$$

Due to the density of the space $\mathscr{D}\left(\mathbb{R}^{3}\right)$ in $W_{-\alpha}^{1}\left(\mathbb{R}^{3}\right)$ and since $\boldsymbol{f}$ belongs to $W_{\alpha+1}^{0}(\Omega)$, we deduce that $\boldsymbol{F}$ belongs to $W_{\alpha}^{-1}\left(\mathbb{R}^{3}\right)$. In order to apply Proposition 2.2 , the main point is now to show that $\boldsymbol{F}$ is orthogonal to harmonic polynomials of $W_{-\alpha}^{1}\left(\mathbb{R}^{3}\right)$, namely to the space $\mathscr{P}_{\alpha-1}^{\Delta}$. So let $\boldsymbol{\lambda}$ be in $\mathscr{P}_{\alpha-1}^{\Delta}$ and in view of the above computations, we need to prove that

$$
\langle\boldsymbol{F}, \boldsymbol{\lambda}\rangle_{W_{\alpha}^{-1}\left(\mathbb{R}^{3}\right) \times W_{-\alpha}^{1}\left(\mathbb{R}^{3}\right)}=\int_{\Omega} \boldsymbol{f} \cdot \boldsymbol{\lambda} \mathrm{d} \boldsymbol{x}+\langle\boldsymbol{h} \times \boldsymbol{n}, \boldsymbol{\lambda}\rangle_{\Gamma}+\langle\boldsymbol{\lambda} \cdot \boldsymbol{n}, \chi\rangle_{\Gamma}-\left\langle\boldsymbol{\lambda} \cdot \boldsymbol{n}, \operatorname{div} \boldsymbol{u}^{\prime}\right\rangle_{\Gamma}-\left\langle\operatorname{curl} \boldsymbol{u}^{\prime} \times \boldsymbol{n}, \boldsymbol{\lambda}\right\rangle_{\Gamma}=0
$$

But, on the one hand, computations in $\Omega^{\prime}$ using (2.13), (2.14), the fact that $\boldsymbol{u}=\boldsymbol{u}^{\prime}$ on $\Gamma$ and so $\boldsymbol{u} \cdot \boldsymbol{n}=$ $\boldsymbol{u} \cdot \boldsymbol{n}=0$ on $\Gamma$, show that

$$
\begin{aligned}
0 & =\int_{\Omega^{\prime}} \boldsymbol{\lambda} \cdot \Delta \boldsymbol{u}^{\prime} \mathrm{d} \boldsymbol{x} \\
& =-\left\langle\operatorname{curl} \boldsymbol{u}^{\prime} \times \boldsymbol{n}, \boldsymbol{\lambda}\right\rangle_{\Gamma}+\left\langle\operatorname{curl} \boldsymbol{\lambda} \times \boldsymbol{n}, \boldsymbol{u}^{\prime}\right\rangle_{\Gamma}-\left\langle\boldsymbol{\lambda} \cdot \boldsymbol{n}, \operatorname{div} \boldsymbol{u}^{\prime}\right\rangle_{\Gamma} \\
& =-\left\langle\operatorname{curl} \boldsymbol{u}^{\prime} \times \boldsymbol{n}, \boldsymbol{\lambda}\right\rangle_{\Gamma}+\langle\operatorname{curl} \boldsymbol{\lambda} \times \boldsymbol{n}, \boldsymbol{u}\rangle_{\Gamma}-\left\langle\boldsymbol{\lambda} \cdot \boldsymbol{n}, \operatorname{div} \boldsymbol{u}^{\prime}\right\rangle_{\Gamma}
\end{aligned}
$$


which implies that

$$
-\left\langle\operatorname{curl} \boldsymbol{u}^{\prime} \times \boldsymbol{n}, \boldsymbol{\lambda}\right\rangle_{\Gamma}-\left\langle\boldsymbol{\lambda} \cdot \boldsymbol{n}, \operatorname{div} \boldsymbol{u}^{\prime}\right\rangle_{\Gamma}=-\langle\operatorname{curl} \boldsymbol{\lambda} \times \boldsymbol{n}, \boldsymbol{u}\rangle_{\Gamma}
$$

On the other hand, similar computations in $\Omega$ show that

$$
\begin{aligned}
\int_{\Omega} \boldsymbol{f} \cdot \boldsymbol{\lambda} \mathrm{d} \boldsymbol{x} & =-\int_{\Omega} \Delta \boldsymbol{u} \cdot \boldsymbol{\lambda} \mathrm{d} \boldsymbol{x} \\
& =-\int_{\Omega} \boldsymbol{u} \cdot \Delta \boldsymbol{\lambda} \mathrm{d} \boldsymbol{x}+\langle\operatorname{curl} \boldsymbol{\lambda} \times \boldsymbol{n}, \boldsymbol{u}\rangle_{\Gamma}-\langle\boldsymbol{h} \times \boldsymbol{n}, \boldsymbol{\lambda}\rangle_{\Gamma}-\langle\boldsymbol{\lambda} \cdot \boldsymbol{n}, \chi\rangle_{\Gamma},
\end{aligned}
$$

which implies that

$$
\int_{\Omega} \boldsymbol{f} \cdot \boldsymbol{\lambda} \mathrm{d} \boldsymbol{x}=\langle\operatorname{curl} \boldsymbol{\lambda} \times \boldsymbol{n}, \boldsymbol{u}\rangle_{\Gamma}-\langle\boldsymbol{h} \times \boldsymbol{n}, \boldsymbol{\lambda}\rangle_{\Gamma}-\langle\boldsymbol{\lambda} \cdot \boldsymbol{n}, \chi\rangle_{\Gamma} .
$$

Combining (3.55) and (3.56) allow to prove (3.54). It follows from Proposition 2.2 that there exists $\widetilde{\boldsymbol{v}} \in W_{\alpha}^{1}\left(\mathbb{R}^{3}\right)$ satisfying

$$
\Delta \widetilde{\boldsymbol{v}}=\Delta \widetilde{\boldsymbol{u}} \text { in } \mathbb{R}^{3} .
$$

As a result, since $\alpha>0$, the difference $\widetilde{\boldsymbol{u}}-\widetilde{\boldsymbol{v}} \in W_{0}^{1}\left(\mathbb{R}^{3}\right)$ is a harmonic polynomials and therefore $\widetilde{\boldsymbol{u}}=\widetilde{\boldsymbol{v}}$ which in turn shows that $\boldsymbol{u} \in W_{\alpha}^{1}(\Omega)$.

- THE CASE $g \neq 0$. Let $w$ be in $W_{\alpha+1}^{3}(\Omega) \operatorname{such} \nabla w \cdot \boldsymbol{n}=g$. Next consider the problem:

$$
\left\{\begin{array}{l}
-\Delta \boldsymbol{z}=\boldsymbol{f}+\Delta \nabla w \text { and } \operatorname{div} \boldsymbol{z}=\chi-\Delta w \text { in } \Omega, \\
\boldsymbol{z} \cdot \boldsymbol{n}=\mathbf{0} \text { and } \operatorname{curl} \boldsymbol{z} \times \boldsymbol{n}=\boldsymbol{h} \times \boldsymbol{n} \text { on } \Gamma .
\end{array}\right.
$$

According to the previous case, problem (3.57) has a solution $z \in W_{\alpha}^{1}(\Omega)$ if the following conditions are satisfied:

$$
\begin{aligned}
& \operatorname{div}(\boldsymbol{f}+\Delta \nabla w+\nabla \chi-\nabla \Delta w)=0 \quad \text { in } \quad \Omega, \\
& (\boldsymbol{f}+\Delta \nabla w+\nabla \chi-\nabla \Delta w) \cdot \boldsymbol{n}=\operatorname{div}_{\Gamma}(\boldsymbol{h} \times \boldsymbol{n}) \quad \text { on } \quad \Gamma, \\
& \int_{\Omega}(\boldsymbol{f}+\Delta \nabla w) \cdot \boldsymbol{\varphi} \mathrm{d} \boldsymbol{x}+\langle\boldsymbol{h} \times \boldsymbol{n}, \boldsymbol{\varphi}\rangle_{\Gamma}=0, \quad \forall \boldsymbol{\varphi} \in Y_{-\alpha-1, T}(\Omega) .
\end{aligned}
$$

Conditions (3.58a) and (3.58b) follow immediately from (3.37) and (3.38) respectively. Besides, if $\boldsymbol{\varphi} \in$ $Y_{-\alpha-1, T}(\Omega)$, then using (2.13), we clearly have

$$
\int_{\Omega} \Delta \nabla w \cdot \boldsymbol{\varphi} \mathrm{d} \boldsymbol{x}=0
$$

Then (3.58c) follows from (3.39). We deduce that there exists $z \in W_{\alpha}^{1}(\Omega)$ satisfying (3.57) and $\boldsymbol{u}=$ $z+\nabla w \in W_{\alpha}^{1}(\Omega)$ is the required solution of $\left(\mathscr{E}_{T}\right)$. 


\section{Exterior Stokes problem with Non Standard Boundary Conditions}

In this section, we study the following exterior Stokes problem: given $\boldsymbol{f}, \boldsymbol{h}, \chi$ and $g$, we look for a pair $(\boldsymbol{u}, \pi)$ that satisfies:

$$
\left\{\begin{array}{l}
-\Delta \boldsymbol{u}+\nabla \pi=\boldsymbol{f} \text { and } \operatorname{div} \boldsymbol{u}=\chi \text { in } \Omega, \\
\boldsymbol{u} \cdot \boldsymbol{n}=g \text { and } \operatorname{curl} \boldsymbol{u} \times \boldsymbol{n}=\boldsymbol{h} \times \boldsymbol{n} \text { on } \Gamma .
\end{array}\right.
$$

We first recall the result on the existence and the regularity of the variational solution of $\left(\mathscr{S}_{T}\right)$ proved in [5] (see Corollary 5.2 and Theorem 6.1).

Theorem 4.1. Assume that $\boldsymbol{f} \in H_{1}^{-1}(\operatorname{div}, \Omega), \chi \in L^{2}(\Omega), g \in H^{1 / 2}(\Gamma)$ and $\boldsymbol{h} \in H^{-1 / 2}(\Gamma)$. Then problem $\left(\mathscr{S}_{T}\right)$ has a unique solution $(\boldsymbol{u}, \pi) \in W_{0}^{1}(\Omega) \times L^{2}(\Omega)$ and there exists a constant $C$ such that:

$$
\|\boldsymbol{u}\|_{W_{0}^{1}(\Omega)}+\|\pi\|_{L^{2}(\Omega)} \leqslant C\left(\|\boldsymbol{f}\|_{H_{1}^{-1}(\operatorname{div}, \Omega)}+\|\boldsymbol{h} \times \boldsymbol{n}\|_{H^{-1 / 2}(\Gamma)}+\|\chi\|_{L^{2}(\Omega)}+\|g\|_{H^{1 / 2}(\Gamma)}\right) .
$$

Moreover if $\boldsymbol{f} \in W_{1}^{0}(\Omega), \chi \in W_{1}^{1}(\Omega), g \in H^{3 / 2}(\Gamma)$ and $\boldsymbol{h} \in H^{1 / 2}(\Gamma)$, then $(\boldsymbol{u}, \pi) \in W_{1}^{2}(\Omega) \times W_{1}^{1}(\Omega)$.

\subsection{Strong solutions}

As for problem $\left(\mathscr{E}_{T}\right)$, we are interested here in looking for strong solution $(\boldsymbol{u}, \pi) \in W_{\alpha+1}^{2}(\Omega) \times W_{\alpha+1}^{1}(\Omega)$ for any $\alpha \in \mathbb{Z}$. Let us first give a characterization of the kernel $\mathscr{S}_{\alpha}(\Omega)$ defined by:

$$
\begin{aligned}
\mathscr{S}_{\alpha}(\Omega)= & \left\{(\boldsymbol{u}, \pi) \in W_{\alpha+1}^{2}(\Omega) \times W_{\alpha+1}^{1}(\Omega),-\Delta \boldsymbol{u}+\nabla \pi=\mathbf{0}, \operatorname{div} \boldsymbol{u}=0 \text { in } \Omega\right. \\
& \boldsymbol{u} \cdot \boldsymbol{n}=0 \text { and curl } \boldsymbol{u} \times \boldsymbol{n}=\mathbf{0} \text { on } \Gamma\} .
\end{aligned}
$$

For all $\alpha \in \mathbb{Z}$, we introduce the following space:

$$
N_{\alpha}=\left\{(\boldsymbol{\lambda}, \mu) \in \mathscr{P}_{\alpha} \times \mathscr{P}_{\alpha-1}^{\Delta} ; \operatorname{div} \boldsymbol{\lambda}=0 \text { and }-\Delta \boldsymbol{\lambda}+\nabla \mu=\mathbf{0} \text { in } \mathbb{R}^{3}\right\}
$$

that is the null space of the Stokes operator in the whole space $\mathbb{R}^{3}$. We recall that $N_{\alpha}=\{(\mathbf{0}, 0)\}$, when $\alpha<0$ and $N_{0}=\mathscr{P}_{0} \times\{0\}$. The reader can refer to [16] for the proof and for more details.

Proposition 4.2. Assume that $\alpha \in \mathbb{Z}$.

1. If $\alpha \geqslant 0$, then $\mathscr{S}_{\alpha}(\Omega)=\{(\mathbf{0}, 0)\}$.

2. If $\alpha<0$, then $\mathscr{S}_{\alpha}(\Omega)=\left\{(\boldsymbol{v}(\boldsymbol{\lambda})+\boldsymbol{\lambda}, \theta(\boldsymbol{\lambda})+\mu) ;(\boldsymbol{\lambda}, \mu) \in N_{-\alpha-1}\right\}$, where the pair $(\boldsymbol{v}(\boldsymbol{\lambda}), \theta(\boldsymbol{\lambda})) \in W_{1}^{2}(\Omega) \times$ $W_{1}^{1}(\Omega)$ is the unique solution of

$$
\left\{\begin{array}{l}
-\Delta \boldsymbol{v}(\boldsymbol{\lambda})+\nabla \theta(\boldsymbol{\lambda})=\mathbf{0} \quad \text { and } \quad \operatorname{div} \boldsymbol{v}(\boldsymbol{\lambda})=0 \quad \text { in } \Omega \\
(\boldsymbol{\nu}(\boldsymbol{\lambda})+\boldsymbol{\lambda}) \cdot \boldsymbol{n}=0 \quad \text { and } \quad(\operatorname{curl} \boldsymbol{v}(\boldsymbol{\lambda})+\operatorname{curl} \boldsymbol{\lambda}) \times \boldsymbol{n}=\mathbf{0} \quad \text { on } \Gamma
\end{array}\right.
$$


Proof.

1. Because $\alpha \geqslant 0$, we clearly have $\mathscr{S}_{\alpha}(\Omega) \subset \mathscr{S}_{0}(\Omega)$. But Theorem 4.1 implies that $\mathscr{S}_{0}(\Omega)=\{(\mathbf{0}, 0)\}$.

2. Let $(\boldsymbol{u}, \pi) \in \mathscr{S}_{\alpha}(\Omega)$ and denote by $\tilde{\boldsymbol{u}}$ and $\tilde{\pi}$ their extensions to $\mathbb{R}^{3}$ that belong to $W_{\alpha+1}^{2}\left(\mathbb{R}^{3}\right) \times W_{\alpha+1}^{1}\left(\mathbb{R}^{3}\right)$. Define

$$
\boldsymbol{F}=-\Delta \tilde{\boldsymbol{u}}+\nabla \tilde{\pi} \text { and } H=\operatorname{div} \tilde{\boldsymbol{u}} \text { in } \mathbb{R}^{3} .
$$

Then clearly the pair $(\boldsymbol{F}, H)$ belongs to $W_{\alpha+1}^{0}\left(\mathbb{R}^{3}\right) \times W_{\alpha+1}^{1}\left(\mathbb{R}^{3}\right)$ and has a compact support. Thanks to [16, Theorem 4.4], there exists $(z, \theta) \in W_{1}^{2}\left(\mathbb{R}^{3}\right) \times W_{1}^{1}\left(\mathbb{R}^{3}\right)$ satisfying the following Stokes problem:

$$
-\Delta \boldsymbol{z}+\nabla \theta=\boldsymbol{F} \text { and } \operatorname{div} \boldsymbol{z}=H \text { in } \mathbb{R}^{3} .
$$

It follows that

$$
-\Delta(\boldsymbol{z}-\tilde{\boldsymbol{u}})+\nabla(\theta-\tilde{\pi})=\mathbf{0} \text { and } \operatorname{div}(\boldsymbol{z}-\tilde{\boldsymbol{u}})=0 \text { in } \quad \mathbb{R}^{3} .
$$

Because $\alpha<0$, we deduce that $(\boldsymbol{z}-\tilde{\boldsymbol{u}}, \theta-\tilde{\pi})$ belongs to $W_{\alpha+1}^{2}\left(\mathbb{R}^{3}\right) \times W_{\alpha+1}^{1}\left(\mathbb{R}^{3}\right)$. Then thanks again to [16, Theorem 4.4], we deduce that $(\boldsymbol{z}-\tilde{\boldsymbol{u}}, \theta-\tilde{\pi}) \in N_{-\alpha-1}$ which means that there exists $(\boldsymbol{\lambda}, \mu) \in N_{-\alpha-1}$ such that $z-\tilde{\boldsymbol{u}}=\boldsymbol{\lambda}$ and $\theta-\tilde{\boldsymbol{\pi}}=\mu$. Therefore the restriction of $(\boldsymbol{z}, \theta)$ to $\Omega$ belongs to $W_{\alpha+1}^{2}(\Omega) \times W_{\alpha+1}^{1}(\Omega)$ and satisfies (4.60).

We are now ready to state the existence and the uniqueness of strong solution to problem $\left(\mathscr{S}_{T}\right)$.

Theorem 4.3. Let $\alpha \in \mathbb{Z}$ and assume that $\boldsymbol{f} \in W_{\alpha+1}^{0}(\Omega), \chi \in W_{\alpha+1}^{1}(\Omega), g \in H^{3 / 2}(\Gamma)$ and $\boldsymbol{h} \in H^{1 / 2}(\Gamma)$ such that

$$
\forall(\boldsymbol{\xi}, \eta) \in \mathscr{S}_{-\alpha}(\Omega), \quad \int_{\Omega} \boldsymbol{f} \cdot \boldsymbol{\xi} \mathrm{d} \boldsymbol{x}-\int_{\Omega} \chi \eta \mathrm{d} \boldsymbol{x}+\langle\boldsymbol{h} \times \boldsymbol{n}, \boldsymbol{\xi}\rangle_{\Gamma}+\langle g, \eta\rangle_{\Gamma}=0 .
$$

Then, problem $\left(\mathscr{S}_{T}\right)$ has a unique solution $(\boldsymbol{u}, \pi) \in W_{\alpha+1}^{2}(\Omega) \times W_{\alpha+1}^{1}(\Omega) / \mathscr{S}_{\alpha}^{2}(\Omega)$ and we have the following estimate:

$$
\inf _{(\xi, \eta) \in \mathcal{S}_{\alpha}^{2}(\Omega)}\left(\|\boldsymbol{u}+\boldsymbol{\xi}\|_{W_{\alpha+1}^{2}(\Omega)}+\|\pi+\eta\|_{W_{\alpha+1}^{1}(\Omega)}\right) \leqslant C\left(\|\boldsymbol{f}\|_{W_{\alpha+1}^{0}(\Omega)}+\|\chi\|_{W_{\alpha+1}^{1}(\Omega)}+\|g\|_{H^{3 / 2}(\Gamma)}+\|\boldsymbol{h} \times \boldsymbol{n}\|_{H^{1 / 2}(\Gamma)}\right) .
$$

Proof. Observe first that the uniqueness is a straightforward consequence of Proposition 4.2. We now divide the proof of the theorem into two parts dedicated to the compatibility condition and the existence statement.

- Compatibility condition. Let us prove that (4.61) is a necessary condition. Suppose that $(\boldsymbol{u}, \pi) \in$ $W_{\alpha+1}^{2}(\Omega) \times W_{\alpha}^{1}(\Omega)$ is a solution of problem $\left(\mathscr{S}_{T}\right)$. For any $(\xi, \eta) \in \mathscr{D}(\bar{\Omega}) \times \mathscr{D}(\bar{\Omega})$, thanks to $(2.13)$ and (2.14), 
we can write:

$$
\begin{aligned}
\int_{\Omega} \boldsymbol{f} \cdot \boldsymbol{\xi} \mathrm{d} \boldsymbol{x} & =\int_{\Omega}(-\Delta \boldsymbol{u}+\nabla \pi) \cdot \boldsymbol{\xi} \mathrm{d} \boldsymbol{x}=-\int_{\Omega} \Delta \boldsymbol{u} \cdot \boldsymbol{\xi} \mathrm{d} \boldsymbol{x}+\int_{\Omega} \nabla \pi \cdot \boldsymbol{\xi} \mathrm{d} \boldsymbol{x} \\
& =\int_{\Omega}(\operatorname{curl} \operatorname{curl} \boldsymbol{u}-\nabla \chi) \cdot \boldsymbol{\xi} \mathrm{d} \boldsymbol{x}+\int_{\Omega} \nabla \pi \cdot \boldsymbol{\xi} \mathrm{d} \boldsymbol{x} \\
& =-\int_{\Omega} \boldsymbol{u} \cdot \Delta \boldsymbol{\xi} \mathrm{d} \boldsymbol{x}-\int_{\Omega} \pi \operatorname{div} \boldsymbol{\xi} \mathrm{d} \boldsymbol{x}+\langle\boldsymbol{u}, \operatorname{curl} \boldsymbol{\xi} \times \boldsymbol{n}\rangle_{\Gamma}-\langle\boldsymbol{h} \times \boldsymbol{n}, \boldsymbol{\xi}\rangle_{\Gamma}+\langle\chi, \boldsymbol{\xi} \cdot \boldsymbol{n}\rangle_{\Gamma}+\langle\pi, \boldsymbol{\xi} \cdot \boldsymbol{n}\rangle_{\Gamma}
\end{aligned}
$$

and

$$
\int_{\Omega} \chi \eta \mathrm{d} \boldsymbol{x}=-\int_{\Omega} \boldsymbol{u} \cdot \nabla \eta \mathrm{d} \boldsymbol{x}+\langle g, \eta\rangle_{\Gamma}
$$

Then making the difference between these relations allows to obtain that for any $(\boldsymbol{\xi}, \eta) \in \mathscr{D}(\bar{\Omega}) \times \mathscr{D}(\bar{\Omega})$,

$$
\begin{aligned}
\int_{\Omega} \boldsymbol{f} \cdot \boldsymbol{\xi} \mathrm{d} \boldsymbol{x}-\int_{\Omega} \chi \eta \mathrm{d} \boldsymbol{x} & =\int_{\Omega} \boldsymbol{u} \cdot(-\Delta \boldsymbol{\xi}+\nabla \eta) \mathrm{d} \boldsymbol{x}-\int_{\Omega} \pi \operatorname{div} \boldsymbol{\xi} \mathrm{d} \boldsymbol{x}+\langle\boldsymbol{u}, \operatorname{curl} \boldsymbol{\xi} \times \boldsymbol{n}\rangle_{\Gamma}-\langle\boldsymbol{h} \times \boldsymbol{n}, \boldsymbol{\xi}\rangle_{\Gamma} \\
& +\langle\chi, \boldsymbol{\xi} \cdot \boldsymbol{n}\rangle_{\Gamma}+\langle\pi, \boldsymbol{\xi} \cdot \boldsymbol{n}\rangle_{\Gamma}-\langle g, \eta\rangle_{\Gamma} .
\end{aligned}
$$

Since $\mathscr{D}(\bar{\Omega})$ is dense in $W_{\alpha+1}^{2}(\Omega)$ and in $W_{\alpha+1}^{1}(\Omega)$, then the above relation is still valid for any $(\xi, \eta) \in$ $W_{\alpha+1}^{2}(\Omega) \times W_{\alpha+1}^{1}(\Omega)$. In particular, if $(\boldsymbol{\xi}, \eta)$ belongs to $\mathscr{S}_{-\alpha}(\Omega)$, we obtain (4.61).

- EXISTENCE. Let us introduce the following Generalized Neumann problem:

$$
\operatorname{div}(\nabla \pi-\boldsymbol{f}-\nabla \chi)=0 \quad \text { in } \quad \Omega \quad \text { and } \quad(\nabla \pi-\boldsymbol{f}-\nabla \chi) \cdot \boldsymbol{n}=-\operatorname{div}_{\Gamma}(\boldsymbol{h} \times \boldsymbol{n}) \quad \text { on } \quad \Gamma .
$$

In order to use Theorem 3.5 if $\alpha>-1$, we need to prove that the following compatibility condition is satisfied

$$
\forall \varphi \in \mathscr{N}_{-\alpha-1}^{\Delta}(\Omega), \quad-\int_{\Omega}(\boldsymbol{f}+\nabla \chi) \cdot \nabla \varphi \mathrm{d} \boldsymbol{x}=\left\langle-\operatorname{div}_{\Gamma}(\boldsymbol{h} \times \boldsymbol{n}), \varphi\right\rangle_{\Gamma} .
$$

First observe that, thanks to (2.13), we have:

$$
\forall \varphi \in \mathscr{N}_{-\alpha-1}^{\Delta}(\Omega), \quad \int_{\Omega} \nabla \chi \cdot \nabla \varphi \mathrm{d} \boldsymbol{x}=0
$$

Next, owing to Remark 3.4, if $\varphi \in \mathscr{N}_{-\alpha-1}^{\Delta}(\Omega)$, then $\varphi$ also belongs to $W_{-\alpha+1}^{3}(\Omega)$. As a result, $\nabla \varphi \in$ $W_{-\alpha+1}^{2}(\Omega)$ and it follows that $(\nabla \varphi, 0)$ belongs to $\mathscr{S}_{-\alpha}(\Omega)$. Hence, in view of (4.61) we have:

$$
\int_{\Omega} \boldsymbol{f} \cdot \nabla \varphi \mathrm{d} \boldsymbol{x}+\langle\boldsymbol{h} \times \boldsymbol{n}, \nabla \varphi\rangle_{\Gamma}=0
$$

which implies that

$$
-\int_{\Omega} \boldsymbol{f} \cdot \nabla \varphi \mathrm{d} \boldsymbol{x}=\langle\boldsymbol{h} \times \boldsymbol{n}, \nabla \varphi\rangle_{\Gamma}
$$

and (4.63). Then thanks to Theorems 3.2 and 3.5, there exists $\pi \in W_{\alpha+1}^{1}(\Omega)$ satisfying (4.62). As a 
consequence, problem $\left(\mathscr{S}_{T}\right)$ is reduced to

$$
\left\{\begin{array}{l}
-\Delta \boldsymbol{u}=\boldsymbol{f}-\nabla \pi \quad \text { and } \operatorname{div} \boldsymbol{u}=\chi \quad \text { in } \Omega, \\
\boldsymbol{u} \cdot \boldsymbol{n}=g \text { and } \quad \text { curl } \boldsymbol{u} \times \boldsymbol{n}=\boldsymbol{h} \times \boldsymbol{n} \text { on } \Gamma .
\end{array}\right.
$$

Thanks again to Remark 3.4 and the characterization of the space $Y_{-\alpha-1, T}(\Omega)$, if $\boldsymbol{\varphi} \in Y_{-\alpha-1, T}(\Omega)$, then $\boldsymbol{\varphi}$ belongs to $W_{-\alpha+1}^{2}(\Omega)$ and as a consequence, $(\boldsymbol{\varphi}, 0) \in \mathscr{S}_{-\alpha}(\Omega)$ and (4.61) implies that

$$
\int_{\Omega} \boldsymbol{f} \cdot \boldsymbol{\varphi} \mathrm{d} \boldsymbol{x}+\langle\boldsymbol{h} \times \boldsymbol{n}, \boldsymbol{\varphi}\rangle_{\Gamma}=0
$$

Besides, using (2.13), we have

$$
\int_{\Omega} \nabla \pi \cdot \boldsymbol{\varphi} \mathrm{d} \boldsymbol{x}=0
$$

We therefore deduce that

$$
\int_{\Omega}(\boldsymbol{f}-\nabla \pi) \cdot \boldsymbol{\varphi} \mathrm{d} \boldsymbol{x}+\langle\boldsymbol{h} \times \boldsymbol{n}, \boldsymbol{\varphi}\rangle_{\Gamma}=0 .
$$

Thus, thanks to Theorems 3.10 and 3.11, there exists $\boldsymbol{u} \in W_{\alpha+1}^{2}(\Omega)$ satisfying (4.64) and this ends the proof of the theorem.

\subsection{Very weak solutions}

In this subsection, we study the existence and the uniqueness of very weak solution $(\boldsymbol{u}, \pi) \in W_{\alpha}^{0}(\Omega) \times W_{\alpha}^{-1}(\Omega)$, where $\alpha \in \mathbb{Z}$, for the Stokes problem $\left(\mathscr{S}_{T}\right)$. The main idea here relies on the use of a duality argument using the strong solutions obtained in the previous subsection. To that aim, we introduce the following space for any $\alpha \in \mathbb{Z}$ :

$$
T_{\alpha}(\Omega)=\left\{\boldsymbol{v} \in \stackrel{\circ}{H}_{\alpha-2}(\operatorname{div}, \Omega) ; \operatorname{div} \boldsymbol{v} \in \stackrel{\circ}{W}_{\alpha}^{1}(\Omega)\right\},
$$

which is a Hilbert space equipped with the norm

$$
\|\boldsymbol{v}\|_{T_{\alpha}(\Omega)}=\|\boldsymbol{v}\|_{W_{\alpha-2}^{0}(\Omega)}+\|\operatorname{div} \boldsymbol{v}\|_{W_{\alpha}^{1}(\Omega)} .
$$

The next two lemmas give some properties related to the space $T_{\alpha}(\Omega)$. Their proofs are postponed in Section B and Section C.

Lemma 4.4. Assume $\alpha \in \mathbb{Z}$. A distribution $\boldsymbol{f}$ belongs to $\left(T_{\alpha}(\Omega)\right)^{\prime}$ if and only if there exist $\boldsymbol{\phi} \in W_{-\alpha+2}^{0}(\Omega)$ and $f_{0} \in W_{-\alpha}^{-1}(\Omega)$ such that

$$
\forall \boldsymbol{\varphi} \in T_{\alpha}(\Omega), \quad\langle\boldsymbol{f}, \boldsymbol{\varphi}\rangle_{\left(T_{\alpha}(\Omega)\right)^{\prime} \times T_{\alpha}(\Omega)}=\int_{\Omega} \boldsymbol{\phi} \cdot \boldsymbol{\varphi} \mathrm{d} \boldsymbol{x}+\left\langle f_{0}, \operatorname{div} \boldsymbol{\varphi}\right\rangle_{W_{-\alpha}^{-1}(\Omega) \times \stackrel{\circ}{\alpha}_{\alpha}^{1}(\Omega)} .
$$


Moreover, the following estimate holds:

$$
\|\boldsymbol{f}\|_{\left(T_{\alpha}(\Omega)\right)^{\prime}}=\max \left\{\|\boldsymbol{\phi}\|_{W_{-\alpha+2}^{0}(\Omega)},\left\|f_{0}\right\|_{W_{-\alpha}^{-1}(\Omega)}\right\} .
$$

Lemma 4.5. Let $\alpha \in \mathbb{Z}$. Then the following properties hold:

(i) The space $\mathscr{D}(\Omega)$ is dense in $T_{\alpha}(\Omega)$. As a consequence, relation (4.65) implies that

$$
\boldsymbol{f}=\boldsymbol{\phi}+\nabla f_{0}
$$

(ii) For any $\boldsymbol{v} \in T_{\alpha}(\Omega)$ and $\chi \in W_{-\alpha}^{-1}(\Omega)$, we have

$$
\langle\nabla \chi, \boldsymbol{v}\rangle_{\left(T_{\alpha}(\Omega)\right)^{\prime} \times T_{\alpha}(\Omega)}=-\langle\chi, \operatorname{div} \boldsymbol{v}\rangle_{W_{-\alpha}^{-1}(\Omega) \times \stackrel{\leftrightarrow}{W}_{\alpha}^{1}(\Omega)} .
$$

Next, to give a sense to the boundary conditions, we introduce, for any $\alpha \in \mathbb{Z}$, the following space:

$$
H_{\alpha}(\Delta, \Omega)=\left\{\boldsymbol{v} \in W_{-\alpha}^{0}(\Omega) ; \Delta \boldsymbol{v} \in\left(T_{\alpha}(\Omega)\right)^{\prime}\right\}
$$

equipped with the norm:

$$
\|\boldsymbol{v}\|_{H_{\alpha}(\Delta, \Omega)}=\|\boldsymbol{v}\|_{W_{-\alpha}^{0}(\Omega)}+\|\Delta \boldsymbol{v}\|_{\left(T_{\alpha}(\Omega)\right)^{\prime}} .
$$

Lemma 4.6. The space $\mathscr{D}(\bar{\Omega})$ is dense in $H_{\alpha}(\Delta, \Omega)$.

The proof of this lemma is given in Section D. Finally, in order to write a Green formula, we define for $\alpha \in \mathbb{Z}$ :

$$
Z_{\alpha}(\Omega)=\left\{\boldsymbol{v} \in W_{\alpha}^{2}(\Omega) ; \operatorname{div} \boldsymbol{v}=0, \boldsymbol{v} \cdot \boldsymbol{n}=0 \text { and } \operatorname{curl} \boldsymbol{v} \times \boldsymbol{n}=0 \text { on } \Gamma\right\} .
$$

Lemma 4.7. The linear mapping $\gamma: \boldsymbol{u} \longmapsto \operatorname{curl} \boldsymbol{u}_{\mid \Gamma} \times \boldsymbol{n}$ defined on $\mathscr{D}(\bar{\Omega})$ can be extended to a linear and continuous mapping

$$
\gamma: H_{\alpha}(\Delta, \Omega) \longmapsto H^{-3 / 2}(\Gamma)
$$

and we have the following Green formula : for any $\boldsymbol{u} \in H_{\alpha}(\Delta, \Omega)$ and $\boldsymbol{v} \in Z_{\alpha}(\Omega)$

$$
\langle\Delta \boldsymbol{u}, \boldsymbol{v}\rangle_{\left(T_{\alpha}(\Omega)\right)^{\prime} \times T_{\alpha}(\Omega)}=\int_{\Omega} \boldsymbol{u} \cdot \Delta \boldsymbol{v} \mathrm{d} \boldsymbol{x}+\langle\operatorname{curl} \boldsymbol{u} \times \boldsymbol{n}, \boldsymbol{v}\rangle_{H^{-3 / 2}(\Gamma) \times H^{3 / 2}(\Gamma)} .
$$

The proof of this lemma is very similar to that of [6, Lemma 4.14]. But in order to make this paper as selfcontained as possible, we give the proof in Section E.

We now give the definition of a very weak solution of $\left(\mathscr{S}_{T}\right)$.

Definition 4.1. Let $\boldsymbol{f}, \chi, g$ and $\boldsymbol{h}$ be such that

$$
\boldsymbol{f} \in\left(T_{-\alpha}(\Omega)\right)^{\prime}, \quad \chi \in W_{\alpha+1}^{0}(\Omega), \quad g \in H^{-1 / 2}(\Gamma) \quad \text { and } \quad \boldsymbol{h} \times \boldsymbol{n} \in H^{-3 / 2}(\Gamma),
$$


a pair $(\boldsymbol{u}, \pi) \in W_{\alpha}^{0}(\Omega) \times W_{\alpha}^{-1}(\Omega)$ is called very weak solution of $\left(\mathscr{S}_{T}\right)$ if, for any $(\boldsymbol{\varphi}, q) \in Z_{-\alpha}(\Omega) \times W_{-\alpha}^{1}(\Omega)$, the relations

$$
-\int_{\Omega} \boldsymbol{u} \cdot \Delta \boldsymbol{\varphi} \mathrm{d} \boldsymbol{x}-\langle\pi, \operatorname{div} \boldsymbol{\varphi}\rangle_{W_{-\alpha}^{-1}(\Omega) \times \stackrel{1}{W}_{\alpha}^{1}(\Omega)}=\langle\boldsymbol{f}, \boldsymbol{\varphi}\rangle_{\left(T_{-\alpha}(\Omega)\right)^{\prime} \times T_{-\alpha}(\Omega)}+\langle\boldsymbol{h} \times \boldsymbol{n}, \boldsymbol{\varphi}\rangle_{H^{-3 / 2}(\Gamma) \times H^{3 / 2}(\Gamma)}
$$

and

$$
\int_{\Omega} \boldsymbol{u} \cdot \nabla q \mathrm{~d} \boldsymbol{x}=-\int_{\Omega} \chi q \mathrm{~d} \boldsymbol{x}+\langle g, q\rangle_{\Gamma}
$$

are statisfied.

Note that $Z_{-\alpha}(\Omega)$ is included in $T_{-\alpha}(\Omega)$, which insures that all the relations are well defined.

Proposition 4.8. Under the assumptions of Definition 4.1, the two following statements are equivalent:

(A) find $(\boldsymbol{u}, \pi) \in W_{\alpha}^{0}(\Omega) \times W_{\alpha}^{-1}(\Omega)$ a very weak solution of $\left(\mathscr{S}_{T}\right)$

(B) find $(\boldsymbol{u}, \pi) \in W_{\alpha}^{0}(\Omega) \times W_{\alpha}^{-1}(\Omega)$ satisfying $\left(\mathscr{S}_{T}\right)$ in the sense of distributions.

Proof. Let us prove that $(A) \Rightarrow(B)$. So consider $(\boldsymbol{u}, \pi) \in W_{\alpha}^{0}(\Omega) \times W_{\alpha}^{-1}(\Omega)$ a very weak solution of $\left(\mathscr{S}_{T}\right)$. Then thanks to relations (4.70) and (4.71), for any $\boldsymbol{\varphi} \in \mathscr{D}(\Omega)$, we have

$$
-\Delta \boldsymbol{u}+\nabla \pi=\boldsymbol{f} \text { and } \operatorname{div} \boldsymbol{u}=\chi \text { in } \Omega .
$$

Next, using (4.67), it is clear that $\boldsymbol{u}$ belongs to $H_{-\alpha}(\Delta, \Omega)$. Therefore, thanks to (4.68) and (4.69), for any $\boldsymbol{\varphi} \in Z_{-\alpha}(\Omega)$, we can write

$$
\begin{aligned}
-\int_{\Omega} \boldsymbol{u} \cdot \Delta \boldsymbol{\varphi} \mathrm{d} \boldsymbol{u} & =-\langle\Delta \boldsymbol{u}, \boldsymbol{\varphi}\rangle_{\left(T_{-\alpha}(\Omega)\right)^{\prime} \times T_{-\alpha}(\Omega)}+\langle\operatorname{curl} \boldsymbol{u} \times \boldsymbol{n}, \boldsymbol{\varphi}\rangle_{H^{-3 / 2}(\Gamma) \times H^{3 / 2}(\Gamma)} \\
& =\langle\boldsymbol{f}-\nabla \pi, \boldsymbol{\varphi}\rangle_{\left(T_{-\alpha}(\Omega)\right)^{\prime} \times T_{-\alpha}(\Omega)}+\langle\operatorname{curl} \boldsymbol{u} \times \boldsymbol{n}, \boldsymbol{\varphi}\rangle_{H^{-3 / 2}(\Gamma) \times H^{3 / 2}(\Gamma)} \\
& =\langle\boldsymbol{f}, \boldsymbol{\varphi}\rangle_{\left(T_{-\alpha}(\Omega)\right)^{\prime} \times T_{-\alpha}(\Omega)}+\langle\pi, \operatorname{div} \boldsymbol{\varphi}\rangle_{W_{\alpha}^{-1}(\Omega) \times \mathrm{L}_{-\alpha}^{1}(\Omega)}+\langle\operatorname{curl} \boldsymbol{u} \times \boldsymbol{n}, \boldsymbol{\varphi}\rangle_{H^{-3 / 2}(\Gamma) \times H^{3 / 2}(\Gamma)}
\end{aligned}
$$

It follows from (4.70) that for any $\varphi \in Z_{-\alpha}(\Omega)$,

$$
\langle\operatorname{curl} \boldsymbol{u} \times \boldsymbol{n}, \boldsymbol{\varphi}\rangle_{H^{-3 / 2}(\Gamma) \times H^{3 / 2}(\Gamma)}=\langle\boldsymbol{h} \times \boldsymbol{n}, \boldsymbol{\varphi}\rangle_{H^{-3 / 2}(\Gamma) \times H^{3 / 2}(\Gamma)} .
$$

But for any $\boldsymbol{\mu} \in H^{3 / 2}(\Gamma)$, there exists $\boldsymbol{\varphi} \in Z_{-\alpha}(\Omega)$ such that $\boldsymbol{\varphi}=\boldsymbol{\mu}$ on $\Gamma$ (see proof of Lemma 4.7 in Section E). Thus for any $\boldsymbol{\mu} \in H^{3 / 2}(\Gamma)$, we can write

$$
\langle\operatorname{curl} \boldsymbol{u} \times \boldsymbol{n}, \boldsymbol{\mu}\rangle_{H^{-3 / 2}(\Gamma) \times H^{3 / 2}(\Gamma)}=\langle\boldsymbol{h} \times \boldsymbol{n}, \boldsymbol{\mu}\rangle_{H^{-3 / 2}(\Gamma) \times H^{3 / 2}(\Gamma)},
$$

and we deduce that curl $\boldsymbol{u} \times \boldsymbol{n}=\boldsymbol{h} \times \boldsymbol{n}$ on $\Gamma$. Finally, from the equation div $\boldsymbol{u}=\chi$, we deduce that for any $q \in W_{-\alpha}^{-1}(\Omega)$

$$
\langle\boldsymbol{u} \cdot \boldsymbol{n}, q\rangle_{\Gamma}=\langle g, q\rangle_{\Gamma},
$$


which yields $\boldsymbol{u} \cdot \boldsymbol{n}=g$ on $\Gamma$.

Let us now prove $(B) \Rightarrow(A)$. So suppose that $(\boldsymbol{u}, \pi) \in W_{\alpha}^{0}(\Omega) \times W_{\alpha}^{-1}(\Omega)$ satisfies system $\left(\mathscr{S}_{T}\right)$ in the sense of distributions. Then using (4.68), (4.69) and the fact that $\mathscr{D}(\Omega)$ is dense in $T_{-\alpha}(\Omega)$, it is clear that (4.70) holds. Finally, from the equation $\operatorname{div} \boldsymbol{u}=\chi$ and (2.13), we easily deduce (4.71).

Finally, the main result of this part reads as follows:

Theorem 4.9. Let $\boldsymbol{f}, \chi, g$ and $\boldsymbol{h}$ be such that

$$
\boldsymbol{f} \in\left(T_{-\alpha}(\Omega)\right)^{\prime}, \quad \chi \in W_{\alpha+1}^{0}(\Omega), \quad g \in H^{-1 / 2}(\Gamma), \quad \boldsymbol{h} \times \boldsymbol{n} \in H^{-3 / 2}(\Gamma)
$$

and satisfy the necessary condition

$$
\forall(\boldsymbol{\xi}, \eta) \in \mathscr{S}_{-\alpha-1}(\Omega), \quad\langle\boldsymbol{f}, \boldsymbol{\xi}\rangle_{\left(T_{-\alpha}(\Omega)\right)^{\prime} \times T_{-\alpha}(\Omega)}+\langle\boldsymbol{h} \times \boldsymbol{n}, \boldsymbol{\xi}\rangle_{\Gamma}-\int_{\Omega} \chi \eta \mathrm{d} \boldsymbol{x}+\langle g, \eta\rangle_{H^{-1 / 2}(\Gamma) \times H^{1 / 2}(\Gamma)}=0 .
$$

Then, problem $\left(\mathscr{S}_{T}\right)$ has a unique solution $(\boldsymbol{u}, \pi) \in W_{\alpha}^{0}(\Omega) \times W_{\alpha}^{-1}(\Omega) / \mathscr{S}_{\alpha+1}(\Omega)$.

Proof.

Observe that, in view of Proposition 4.8, if the pair $(\boldsymbol{u}, \pi) \in W_{\alpha}^{0}(\Omega) \times W_{\alpha}^{-1}(\Omega)$ is a solution of $\left(\mathscr{S}_{T}\right)$, then for any $(\boldsymbol{\varphi}, q) \in Z_{-\alpha}(\Omega) \times W_{-\alpha}^{1}(\Omega)$, adding (4.70) and (4.71), we have

$$
\begin{aligned}
& \int_{\Omega} \boldsymbol{u} \cdot(-\Delta \boldsymbol{\varphi}+\nabla q) \mathrm{d} \boldsymbol{x}-\langle\pi, \operatorname{div} \boldsymbol{\varphi}\rangle_{W_{-\alpha}^{-1}(\Omega) \times \dot{W}_{\alpha}^{1}(\Omega)} \\
& =\langle\boldsymbol{f}, \boldsymbol{\varphi}\rangle_{\left(T_{-\alpha}(\Omega)\right)^{\prime} \times T_{-\alpha}(\Omega)}+\langle\boldsymbol{h} \times \boldsymbol{n}, \boldsymbol{\varphi}\rangle_{\Gamma}-\int_{\Omega} \chi q \mathrm{~d} \boldsymbol{x}+\langle g, q\rangle_{H^{-1 / 2}(\Gamma) \times H^{1 / 2}(\Gamma)} .
\end{aligned}
$$

In particular, if $(\boldsymbol{\varphi}, q) \in \mathscr{S}_{-\alpha-1}(\Omega)$, we obtain (4.72).

It remains now to look for $(\boldsymbol{u}, \pi) \in W_{\alpha}^{0}(\Omega) \times W_{\alpha}^{-1}(\Omega)$ satisfying (4.73). To that end, we proceed in two steps depending on the datum $g$.

STEP 1: we assume $g=0$. Then let $T$ be the linear form defined by:

$$
\begin{aligned}
T:\left(W_{-\alpha}^{0}(\Omega) \times \stackrel{\circ}{W}_{-\alpha}^{1}(\Omega)\right) \perp \mathscr{S}_{\alpha+1}(\Omega) & \longrightarrow \mathbb{R} \\
(\boldsymbol{w}, \theta) & \longmapsto\langle\boldsymbol{f}, \boldsymbol{\varphi}\rangle_{\left(T_{-\alpha}(\Omega)\right)^{\prime} \times T_{-\alpha}(\Omega)}+\langle\boldsymbol{h} \times \boldsymbol{n}, \boldsymbol{\varphi}\rangle_{\Gamma}-\int_{\Omega} \chi q \mathrm{~d} \boldsymbol{x},
\end{aligned}
$$

where the pair $(\boldsymbol{\varphi}, q) \in W_{-\alpha}^{2}(\Omega) \times W_{-\alpha}^{1}(\Omega)$ is a solution of the following problem (see Theorem 4.3):

$$
\begin{cases}-\Delta \boldsymbol{\varphi}+\nabla q=\boldsymbol{w} & \text { and } \operatorname{div} \boldsymbol{\varphi}=-\theta \text { in } \Omega, \\ \boldsymbol{\varphi} \cdot \boldsymbol{n}=0 \text { and } \operatorname{curl} \boldsymbol{\varphi} \times \boldsymbol{n}=\mathbf{0} \text { on } \Gamma\end{cases}
$$

and satisfies the following estimate:

$$
\inf _{(\xi, \eta) \in \mathscr{S}_{-\alpha-1}(\Omega)}\left(\|\boldsymbol{\varphi}+\boldsymbol{\xi}\|_{W_{-\alpha}^{2}(\Omega)}+\|q+\eta\|_{W_{-\alpha}^{1}(\Omega)}\right) \leqslant C\left(\|\boldsymbol{w}\|_{W_{-\alpha}^{0}(\Omega)}+\|\theta\|_{W_{-\alpha}^{1}(\Omega)}\right) .
$$


Then for any pair $(\boldsymbol{w}, \theta) \in W_{-\alpha}^{0}(\Omega) \times \stackrel{\circ}{W}_{-\alpha}^{1}(\Omega)$ and for any $(\boldsymbol{\xi}, \eta) \in \mathscr{S}_{\alpha+1}(\Omega)$, we can write

$$
\begin{aligned}
|T(\boldsymbol{w}, \theta)| & =\left|\langle\boldsymbol{f}, \boldsymbol{\varphi}\rangle_{\left(T_{-\alpha}(\Omega)\right)^{\prime} \times T_{-\alpha}(\Omega)}+\langle\boldsymbol{h} \times \boldsymbol{n}, \boldsymbol{\varphi}\rangle_{\Gamma}-\int_{\Omega} \chi q \mathrm{~d} \boldsymbol{x}\right| \\
& =\left|\langle\boldsymbol{f}, \boldsymbol{\varphi}+\boldsymbol{\xi}\rangle_{\left(T_{-\alpha}(\Omega)\right)^{\prime} \times T_{-\alpha}(\Omega)}+\langle\boldsymbol{h} \times \boldsymbol{n}, \boldsymbol{\varphi}+\boldsymbol{\xi}\rangle_{\Gamma}-\int_{\Omega} \chi(q+\eta) \mathrm{d} \boldsymbol{x}\right| \\
& \leqslant C\left(\|\boldsymbol{f}\|_{\left(T_{-\alpha}(\Omega)\right)^{\prime}}+\|\chi\|_{W_{\alpha+1}^{0}(\Omega)}+\|\boldsymbol{h} \times \boldsymbol{n}\|_{H^{-3 / 2}(\Gamma)}\right)\left(\|\boldsymbol{w}\|_{W_{\alpha+1}^{0}(\Omega)}+\|\theta\|_{W_{-\alpha}^{1}(\Omega)}\right) .
\end{aligned}
$$

Hence the mapping $T$ defines an element on the dual space of $\left(W_{-\alpha}^{0}(\Omega) \times \stackrel{\circ}{W}_{-\alpha}^{1}(\Omega)\right) \perp \mathscr{S}_{\alpha+1}(\Omega)$. Since the dual space of $\left(W_{-\alpha}^{0}(\Omega) \times \stackrel{\circ}{-\alpha}_{-\alpha}^{1}(\Omega)\right) \perp \mathscr{S}_{\alpha+1}(\Omega)$ is $\left(W_{\alpha}^{0}(\Omega) \times W_{\alpha}^{-1}(\Omega)\right) / \mathscr{S}_{\alpha+1}(\Omega)$, from Riesz' representation theorem, there exists a unique pair $(\boldsymbol{u}, \pi) \in W_{\alpha}^{0}(\Omega) \times W_{\alpha}^{-1}(\Omega) / \mathscr{S}_{\alpha+1}(\Omega)$ satisfying (4.73).

STEP 2: Assume $g \neq 0$. Then according to [17, Lemma 3.7]), there exists $v \in W_{0}^{1}(\Omega)$ satisfying

$$
\Delta v=0 \quad \text { in } \quad \Omega \text { and } \nabla v \cdot \boldsymbol{n}=0 \text { on } \Gamma \text {. }
$$

Let $R$ be a positive real number large enough so that $\overline{\Omega^{\prime}} \subset B_{R}$ and let $\psi \in \mathscr{D}(\bar{\Omega})$ such that $0 \leqslant \psi \leqslant 1$, supp $\psi \subset \bar{\Omega}_{R+1}$ and $\psi=1$ in $\bar{\Omega}_{R}$. Set now $w=v \psi$, then $w$ has obviously a compact support and thus $w$ belongs to $W_{\alpha}^{1}(\Omega)$. Next, $\Delta w=v \Delta \psi+2 \nabla v \cdot \nabla \psi$ has a compact support and thus belongs to $W_{\alpha+1}^{0}(\Omega)$ which is included in $W_{\alpha}^{-1}(\Omega)$ (see (2.9)). Hence, $\Delta(\nabla w)$ belongs to $\left(T_{-\alpha}(\Omega)\right)^{\prime}$. Consider now the following problem:

$$
\left\{\begin{array}{l}
-\Delta \boldsymbol{z}+\nabla \pi=\boldsymbol{f}+\Delta(\nabla w) \text { and } \operatorname{div} \boldsymbol{z}=\chi-\Delta w \text { in } \Omega, \\
\boldsymbol{z} \cdot \boldsymbol{n}=0 \text { and } \operatorname{curl} \boldsymbol{z} \times \boldsymbol{n}=\boldsymbol{h} \times \boldsymbol{n} \text { on } \Gamma .
\end{array}\right.
$$

According to step 1, problem (4.74) has a solution $(z, \pi) \in W_{\alpha}^{0}(\Omega) \times W_{\alpha}^{-1}(\Omega)$ if the following condition is satisfied

$$
\langle\boldsymbol{f}+\Delta(\nabla w), \boldsymbol{\xi}\rangle_{\left(T_{-\alpha}(\Omega)\right)^{\prime} \times T_{-\alpha}(\Omega)}-\int_{\Omega}(\chi-\Delta w) \eta \mathrm{d} \boldsymbol{x}+\langle\boldsymbol{h} \times \boldsymbol{n}, \boldsymbol{\xi}\rangle_{\Gamma}=0, \quad \forall(\boldsymbol{\xi}, \eta) \in S_{-\alpha-1}(\Omega) .
$$

But in view of (4.72), if $(\xi, \eta) \in S_{-\alpha-1}(\Omega)$, then we have

$$
\begin{aligned}
& \langle\boldsymbol{f}+\Delta(\nabla w), \boldsymbol{\xi}\rangle_{\left(T_{-\alpha}(\Omega)\right)^{\prime} \times T_{-\alpha}(\Omega)}-\int_{\Omega}(\chi-\Delta w) \eta \mathrm{d} \boldsymbol{x}+\langle\boldsymbol{h} \times \boldsymbol{n}, \boldsymbol{\xi}\rangle_{\Gamma} \\
& =-\langle g, \eta\rangle_{\Gamma}+\langle\Delta(\nabla w), \boldsymbol{\xi}\rangle_{\left(T_{-\alpha}(\Omega)\right)^{\prime} \times T_{-\alpha}(\Omega)}+\int_{\Omega} \Delta w \eta \mathrm{d} \boldsymbol{x} .
\end{aligned}
$$

Furthermore, owing to (4.68), we clearly have

$$
\langle\Delta(\nabla w), \boldsymbol{\xi}\rangle_{\left(T_{-\alpha}(\Omega)\right)^{\prime} \times T_{-\alpha}(\Omega)}=\langle\nabla(\Delta w), \boldsymbol{\xi}\rangle_{\left(T_{-\alpha}(\Omega)\right)^{\prime} \times T_{-\alpha}(\Omega)}=-\langle\Delta w, \operatorname{div} \boldsymbol{\xi}\rangle_{W_{\alpha}^{-1}(\Omega) \times \dot{W}_{-\alpha}^{1}(\Omega)}=0
$$


Next, using (2.13), we can write

$$
\int_{\Omega} \Delta w \eta \mathrm{d} \boldsymbol{x}=-\int_{\Omega} \nabla w \cdot \nabla \eta \mathrm{d} \boldsymbol{x}+\langle g, \eta\rangle_{\Gamma}
$$

It remains to prove that

$$
\int_{\Omega} \nabla w \cdot \nabla \eta \mathrm{d} \boldsymbol{x}=0
$$

But, using the fact that $(\xi, \eta)$ belongs to $S_{-\alpha-1}(\Omega)$ and using (2.14), we can write

$$
\int_{\Omega} \nabla w \cdot \nabla \eta \mathrm{d} \boldsymbol{x}=\int_{\Omega} \nabla w \cdot \Delta \boldsymbol{\xi} \mathrm{d} \boldsymbol{x}=-\int_{\Omega} \nabla w \cdot \operatorname{curl} \operatorname{curl} \boldsymbol{\xi} \mathrm{d} \boldsymbol{x}
$$

and

$$
0=\langle\operatorname{curl} \boldsymbol{\xi} \times \boldsymbol{n}, \nabla w\rangle_{\Gamma}=\int_{\Omega} \nabla w \cdot \operatorname{curl} \operatorname{curl} \boldsymbol{\xi} \mathrm{d} \boldsymbol{x}
$$

which proves (4.76). This insures the existence of $(z, \pi) \in W_{\alpha}^{0}(\Omega) \times W_{\alpha}^{-1}(\Omega)$ satisfying (4.74) which yields that the pair $(\boldsymbol{u}, \pi)=(\boldsymbol{z}+\nabla w, \pi)$ is the required solution of $\left(\mathscr{S}_{T}\right)$.

\section{Appendices}

\section{A Proof of the statement of Remark 3.9}

Consider the following partition

$$
\begin{aligned}
& \theta_{1}, \theta_{2} \in \mathscr{C}^{\infty}\left(\bar{\Omega}_{R}\right), \quad 0 \leqslant \theta_{1}, \theta_{2} \leqslant 1, \quad \theta_{1}+\theta_{2}=1 \quad \text { in } \bar{\Omega}_{R} \\
& \theta_{1}=1 \quad \text { in } \quad \Omega_{R / 3}, \quad \operatorname{supp} \theta_{1} \subset \Omega_{2 R / 3} .
\end{aligned}
$$

Then we can write $\boldsymbol{u}=\theta_{1} \boldsymbol{u}+\theta_{2} \boldsymbol{u}=\boldsymbol{u}_{1}+\boldsymbol{u}_{2}$. Now it is clear that

$$
-\Delta \boldsymbol{u}_{1}=\boldsymbol{f}_{1} \quad \text { and } \quad \operatorname{div} \boldsymbol{u}_{1}=\chi_{1} \quad \text { in } \quad \Omega_{R}
$$

where $\boldsymbol{f}_{1}=\theta_{1} \boldsymbol{f}-\boldsymbol{u} \Delta \theta_{1}-2 \nabla \boldsymbol{u} \cdot \nabla \theta_{1} \in L^{2}\left(\Omega_{R}\right)$ and $\chi_{1}=\theta \chi+\boldsymbol{u} \cdot \nabla \theta_{1} \in H^{1}\left(\Omega_{R}\right)$. Moreover, under the assumptions on $\theta_{1}$, it is also clear that on the boundaries, we have

$$
\boldsymbol{u}_{1} \cdot \boldsymbol{n}=g \quad, \quad \text { curl } \boldsymbol{u}_{1} \times \boldsymbol{n}=\boldsymbol{h} \times \boldsymbol{n} \quad \text { on } \quad \Gamma \quad \text { and } \quad \boldsymbol{u}_{1} \cdot \boldsymbol{n}=0 \quad, \quad \operatorname{curl} \boldsymbol{u}_{1} \times \boldsymbol{n}=\mathbf{0} \quad \text { on } \partial B_{R} .
$$


Under the assumptions on the data, we deduce that $\boldsymbol{u}_{1}$ that satisfies (A.77)-(A.78) belongs to $H^{2}\left(\Omega_{R}\right.$ ) (see [6, Proposition 4.7]). Similar arguments show that $\boldsymbol{u}_{2}$ satisfies the Dirichlet problem

$$
-\Delta \boldsymbol{u}_{2}=\boldsymbol{f}_{2} \text { in } \Omega_{R}, \quad \boldsymbol{u}_{2}=\mathbf{0} \text { on } \Gamma \text { and } \boldsymbol{u}_{2}=\boldsymbol{a} \text { on } \partial B_{R}
$$

where $\boldsymbol{f}_{2}=\theta_{2} \boldsymbol{f}-\boldsymbol{u} \Delta \theta_{2}-2 \nabla \boldsymbol{u} \cdot \nabla \theta_{2} \in L^{2}\left(\Omega_{R}\right)$. This shows that $\boldsymbol{u}_{2} \in H^{2}\left(\Omega_{R}\right)$ (see for instance [19] or [23]).

\section{B Proof of Lemma 4.4}

Let $\boldsymbol{f} \in\left(T_{\alpha}(\Omega)\right)^{\prime}$ and consider the space $E=W_{\alpha-2}^{0}(\Omega) \times \stackrel{\circ}{W}_{\alpha}^{1}(\Omega)$ equipped with the norm

$$
\forall \boldsymbol{h}=\left(\boldsymbol{h}_{0}, h_{1}\right) \in E, \quad\|\boldsymbol{h}\|_{E}=\left\|\boldsymbol{h}_{0}\right\|_{W_{\alpha-2}^{0}(\Omega)}+\left\|h_{1}\right\|_{W_{\alpha}^{1}(\Omega)} .
$$

The mapping $L: \boldsymbol{\varphi} \in T_{\alpha}(\Omega) \mapsto(\boldsymbol{\varphi}, \operatorname{div} \boldsymbol{\varphi}) \in E$ is an isometric mapping. Set $G=L\left(T_{\alpha}(\Omega)\right)$ and $S=L^{-1}: G \mapsto T_{\alpha}(\Omega)$, then the linear mapping defined by

$$
\boldsymbol{h} \in G \mapsto\langle\boldsymbol{f}, S \boldsymbol{h}\rangle_{\left(T_{\alpha}(\Omega)\right)^{\prime} \times T_{\alpha}(\Omega)}
$$

is linear and continuous on $G$. Therefore thanks to Hahn-Banach theorem, there exists an extension $\Pi$ of $L$ which is linear and continuous on $E$ satisfying $\|\Pi\|_{E^{\prime}}=\|\boldsymbol{f}\|_{\left(T_{\alpha}(\Omega)\right)^{\prime}}$. From the Riesz's Representation theorem, there exists $\boldsymbol{\phi} \in W_{-\alpha+2}^{0}(\Omega)$ and $f_{0} \in W_{-\alpha}^{-1}(\Omega)$ such that for any $\boldsymbol{h}=\left(\boldsymbol{h}_{0}, h_{1}\right) \in E$,

$$
\begin{aligned}
\langle\Pi, \boldsymbol{h}\rangle_{E^{\prime} \times E} & =\left\langle\boldsymbol{\phi}, \boldsymbol{h}_{0}\right\rangle_{W_{-\alpha+2}^{0}(\Omega) \times W_{\alpha-2}^{0}(\Omega)}+\left\langle f_{0}, h_{1}\right\rangle_{W_{-\alpha}^{-1}(\Omega) \times \stackrel{W}{W}_{\alpha}^{1}(\Omega)} \\
& =\int_{\Omega} \boldsymbol{\phi} \cdot \boldsymbol{h}_{0} \mathrm{~d} \boldsymbol{x}+\left\langle f_{0}, h_{1}\right\rangle_{W_{-\alpha}^{-1}(\Omega) \times \stackrel{\mathrm{W}}{\alpha}_{\alpha}^{1}(\Omega)}
\end{aligned}
$$

and it is clear that $\|\Pi\|_{E^{\prime}}=\max \left\{\|\boldsymbol{\phi}\|_{W_{-\alpha+2}^{0}(\Omega)},\left\|f_{0}\right\|_{W_{-\alpha}^{-1}(\Omega)}\right\}$.

\section{Proof of Lemma 4.5}

(i) Let $\ell \in\left(T_{\alpha}(\Omega)\right)^{\prime}$ such that

$$
\langle\boldsymbol{\ell}, \boldsymbol{\varphi}\rangle_{\left(T_{\alpha}(\Omega)\right)^{\prime} \times T_{\alpha}(\Omega)}=0, \quad \forall \boldsymbol{\varphi} \in \mathscr{D}(\Omega) .
$$

Thanks to Lemma 4.4, there exist $\phi \in W_{-\alpha+2}^{0}(\Omega)$ and $f_{0} \in W_{-\alpha}^{-1}(\Omega)$ such that:

$$
\langle\boldsymbol{\ell}, \boldsymbol{\varphi}\rangle_{\left(T_{\alpha}(\Omega)\right)^{\prime} \times T_{\alpha}(\Omega)}=\int_{\Omega} \boldsymbol{\phi} \cdot \boldsymbol{\varphi} \mathrm{d} \boldsymbol{x}+\left\langle f_{0}, \operatorname{div} \boldsymbol{\varphi}\right\rangle_{W_{-\alpha}^{-1}(\Omega) \times \stackrel{\mathrm{W}}{\alpha}^{1}(\Omega)} .
$$

As a consequence

$$
\boldsymbol{\phi}-\nabla f_{0}=0 \text { in } \mathscr{D}^{\prime}(\Omega)
$$

and this implies that $\nabla f_{0}=\boldsymbol{\phi} \in W_{-\alpha+2}^{0}(\Omega)$. It follows that (see [17, Theorem 2.7]) if $\alpha \geqslant 3, f_{0}$ belongs to $W_{-\alpha+2}^{1}(\Omega)$ and if $\alpha \leqslant 2$, there exist a unique real number $c$ and a unique $s \in W_{-\alpha+2}^{1}(\Omega)$ such that $f_{0}=$ 
$s+c$. But because we have the embeddings $W_{-\alpha+2}^{1}(\Omega) \hookrightarrow W_{-\alpha+1}^{0}(\Omega) \hookrightarrow W_{-\alpha}^{-1}(\Omega)$, then $s$ also belongs to $W_{-\alpha}^{-1}(\Omega)$ which in turn implies that $c$ belongs to $W_{-\alpha}^{-1}(\Omega)$. Since $\alpha \leqslant 2$, the space $W_{-\alpha}^{-1}(\Omega)$ does not contain polynomial functions and thus $c=0$. It follows that $f_{0}=s$ also belongs to $W_{-\alpha+2}^{1}(\Omega)$ for $\alpha \leqslant 2$. Therefore, for any $\boldsymbol{v} \in T_{\alpha}(\Omega)$, we can write:

$$
\begin{aligned}
\langle\boldsymbol{\ell}, \boldsymbol{v}\rangle_{\left(T_{\alpha}(\Omega)\right)^{\prime} \times T_{\alpha}(\Omega)} & =\int_{\Omega} \boldsymbol{\phi} \cdot \boldsymbol{v} \mathrm{d} \boldsymbol{x}+\left\langle f_{0}, \operatorname{div} \boldsymbol{v}\right\rangle_{W_{-\alpha}^{-1}(\Omega) \times \dot{W}_{\alpha}^{1}(\Omega)} \\
& =\int_{\Omega} \boldsymbol{\phi} \cdot \boldsymbol{v} \mathrm{d} \boldsymbol{x}+\int_{\Omega} f_{0} \operatorname{div} \boldsymbol{v} \mathrm{d} \boldsymbol{x} \\
& =\int_{\Omega} \nabla f_{0} \cdot \boldsymbol{v} \mathrm{d} \boldsymbol{x}+\int_{\Omega} f_{0} \operatorname{div} \boldsymbol{v} \mathrm{d} \boldsymbol{x} .
\end{aligned}
$$

Finally, because $\boldsymbol{v} \in \stackrel{\circ}{H}_{\alpha-2}(\operatorname{div}, \Omega)$ and $f_{0} \in W_{-\alpha+2}^{1}(\Omega)$, then one can use (2.13) to the first integral of the last relation to obtain

$$
\langle\boldsymbol{\ell}, \boldsymbol{v}\rangle_{\left(T_{\alpha}(\Omega)\right)^{\prime} \times T_{\alpha}(\Omega)}=0, \quad \forall \boldsymbol{v} \in T_{\alpha}(\Omega)
$$

which ends the proof.

(ii) Due to the density of $\mathscr{D}(\Omega)$ in $T_{\alpha}(\Omega)$, then the Green Formula (4.68) clearly holds.

\section{Proof of Lemma 4.6}

Proof. Let $\boldsymbol{\ell} \in\left(H_{\alpha}(\Delta, \Omega)\right)^{\prime}$ such that

$$
\langle\boldsymbol{\ell}, \boldsymbol{v}\rangle_{\left(H_{\alpha}(\Delta, \Omega)\right)^{\prime} \times H_{\alpha}(\Delta, \Omega)}=0, \quad \forall \boldsymbol{v} \in \mathscr{D}(\bar{\Omega}) .
$$

Proceeding as in the proof of Lemma 4.4, we can show that there exists $(\boldsymbol{f}, \boldsymbol{g}) \in W_{\alpha}^{0}(\Omega) \times T_{\alpha}(\Omega)$ such that for any $\boldsymbol{v} \in H_{\alpha}(\Delta, \Omega)$,

$$
\langle\boldsymbol{\ell}, \boldsymbol{v}\rangle_{\left(H_{\alpha}(\Delta, \Omega)\right)^{\prime} \times H_{\alpha}(\Delta, \Omega)}=\int_{\Omega} \boldsymbol{f} \cdot \boldsymbol{v} \mathrm{d} \boldsymbol{x}+\langle\Delta \boldsymbol{v}, \boldsymbol{g}\rangle_{\left(T_{\alpha}(\Omega)\right)^{\prime} \times T_{\alpha}(\Omega)} .
$$

Now, it follows from (D.79), that $\ell=\mathbf{0}$ on $\mathscr{D}(\Omega)$ and thus

$$
\boldsymbol{f}+\Delta \boldsymbol{g}=\mathbf{0} \quad \text { in } \quad \mathscr{D}^{\prime}(\Omega)
$$

Let $\widetilde{\boldsymbol{f}}$ be the extension by zero of $\boldsymbol{f}$ that belongs to $W_{\alpha}^{0}\left(\mathbb{R}^{3}\right)$ and $\widetilde{\boldsymbol{g}}$ be the extension by zero of $\boldsymbol{g}$ that belongs to $H_{\alpha-2}\left(\operatorname{div}, \mathbb{R}^{3}\right)$. Then thanks to (D.79), for any $\boldsymbol{\varphi} \in \mathscr{D}\left(\mathbb{R}^{3}\right)$, we have

$$
\int_{\mathbb{R}^{3}} \tilde{\boldsymbol{f}} \cdot \boldsymbol{\varphi} \mathrm{d} \boldsymbol{x}+\int_{\mathbb{R}^{3}} \widetilde{\boldsymbol{g}} \cdot \Delta \boldsymbol{\varphi} \mathrm{d} \boldsymbol{x}=\int_{\Omega} \boldsymbol{f} \cdot \boldsymbol{\varphi} \mathrm{d} \boldsymbol{x}+\int_{\Omega} \boldsymbol{g} \cdot \Delta \boldsymbol{\varphi} \mathrm{d} \boldsymbol{x}=0
$$

Which yields

$$
-\Delta \widetilde{\boldsymbol{g}}=\widetilde{\boldsymbol{f}} \text { in } \mathscr{D}^{\prime}\left(\mathbb{R}^{3}\right)
$$


As a consequence, $\Delta \widetilde{\mathbf{g}}$ belongs to $W_{\alpha}^{0}\left(\mathbb{R}^{3}\right)$ and, due to the density of $\mathscr{D}\left(\mathbb{R}^{3}\right)$ in $W_{-\alpha+2}^{2}\left(\mathbb{R}^{3}\right)$, we have

$$
\langle\Delta \widetilde{\mathbf{g}}, \boldsymbol{\varphi}\rangle_{W_{\alpha}^{0}\left(\mathbb{R}^{3}\right) \times W_{-\alpha}^{0}\left(\mathbb{R}^{3}\right)}=\langle\widetilde{\mathbf{g}}, \Delta \boldsymbol{\varphi}\rangle_{W_{\alpha-2}^{0}\left(\mathbb{R}^{3}\right) \times W_{-\alpha+2}^{0}\left(\mathbb{R}^{3}\right)}, \quad \forall \boldsymbol{\varphi} \in W_{-\alpha+2}^{2}\left(\mathbb{R}^{3}\right) .
$$

In particular, this shows that $\Delta \widetilde{\mathbf{g}} \in W_{\alpha}^{0}\left(\mathbb{R}^{3}\right)$ is orthogonal to polynomials of $\mathscr{P}_{\alpha-2}^{\Delta}$. Then, according to Proposition 2.2 there exists $\boldsymbol{\lambda} \in W_{\alpha}^{2}\left(\mathbb{R}^{3}\right)$ such that $-\Delta \boldsymbol{\lambda}=\Delta \widetilde{\mathbf{g}}$. Thus the harmonic function $\boldsymbol{\lambda}+\widetilde{\mathbf{g}}$ belongs to $W_{\alpha}^{2}\left(\mathbb{R}^{3}\right)+W_{\alpha-2}^{0}\left(\mathbb{R}^{3}\right) \subset W_{\alpha-2}^{0}\left(\mathbb{R}^{3}\right)$. Then there exists a polynomial $\boldsymbol{K} \in \mathscr{P}_{-\alpha}^{\Delta} \subset W_{\alpha}^{2}\left(\mathbb{R}^{3}\right)$ satisfying $\widetilde{\boldsymbol{g}}=\boldsymbol{K}-\boldsymbol{\lambda} \in$

$W_{\alpha}^{2}\left(\mathbb{R}^{3}\right)$. Hence, because $\boldsymbol{g} \in W_{\alpha}^{2}(\Omega)$, we deduce that $\boldsymbol{g} \in \stackrel{\circ}{W}_{\alpha}^{2}(\Omega)$. Now, due to the density of $\mathscr{D}(\Omega)$ in $\mathfrak{W}_{\alpha}^{2}(\Omega)$, there exists a sequence $\left(\boldsymbol{g}_{k}\right)_{k \in \mathbb{Z}} \subset \mathscr{D}(\Omega)$ such that $\boldsymbol{g}_{k} \rightarrow \boldsymbol{g}$ in $W_{\alpha}^{2}(\Omega)$ as $k \rightarrow \infty$. Consequently $\operatorname{div} \boldsymbol{g}_{k} \rightarrow \operatorname{div} \boldsymbol{g}$ in $W_{\alpha}^{1}(\Omega)$ which shows that $\boldsymbol{g}_{k} \rightarrow \boldsymbol{g}$ in $T_{\alpha}(\Omega)$. Thus for any $\boldsymbol{v} \in H_{\alpha}(\Delta, \Omega)$, we have

$$
\begin{aligned}
\langle\boldsymbol{\ell}, \boldsymbol{v}\rangle_{\left(H_{\alpha}(\Delta, \Omega)\right)^{\prime} \times H_{\alpha}(\Delta, \Omega)} & =\int_{\Omega} \boldsymbol{f} \cdot \boldsymbol{v} \mathrm{d} \boldsymbol{x}+\langle\Delta \boldsymbol{v}, \boldsymbol{g}\rangle_{\left(T_{\alpha}(\Omega)\right)^{\prime} \times T_{\alpha}(\Omega)} \\
& =-\int_{\Omega} \Delta \boldsymbol{g} \cdot \boldsymbol{v} \mathrm{d} \boldsymbol{x}+\langle\Delta \boldsymbol{v}, \boldsymbol{g}\rangle_{\left(T_{\alpha}(\Omega)\right)^{\prime} \times T_{\alpha}(\Omega)} \\
& =\lim _{k \rightarrow \infty}\left(-\int_{\Omega} \Delta \boldsymbol{g}_{k} \cdot \boldsymbol{v} \mathrm{d} \boldsymbol{x}+\left\langle\Delta \boldsymbol{v}, \boldsymbol{g}_{k}\right\rangle_{\left(T_{\alpha}(\Omega)\right)^{\prime} \times T_{\alpha}(\Omega)}\right) \\
& =\lim _{k \rightarrow \infty}\left(-\int_{\Omega} \Delta \boldsymbol{g}_{k} \cdot \boldsymbol{v} \mathrm{d} \boldsymbol{x}+\int_{\Omega} \boldsymbol{v} \cdot \Delta \boldsymbol{g}_{k} \mathrm{~d} \boldsymbol{x}\right) \\
& =0 .
\end{aligned}
$$

\section{E Proof of Lemma 4.7}

Let us first recall, that for any given function $\boldsymbol{v}$ defined on $\Gamma$, we denote by $\boldsymbol{v}_{\boldsymbol{\tau}}$, the tangential component of $\boldsymbol{v}$ on $\Gamma$. Let now $\boldsymbol{x}$ be a point on $\Gamma$ and $\mathcal{V}$ be a neighbourhood of $\boldsymbol{x}$ in $\Gamma$ such that there exists two family of curves of class $\mathscr{C}^{2}$ on $\mathcal{V}$. The lengths $\ell_{1}$ and $\ell_{2}$ along each families are natural system of coordinates in $\mathcal{V}$. Let $\boldsymbol{\tau}_{1}$ and $\boldsymbol{\tau}_{2}$ the unit tangent vectors to each family of curves. We therefore have $\boldsymbol{v}_{\boldsymbol{\tau}}=\left(\boldsymbol{v} \cdot \boldsymbol{\tau}_{1}\right) \boldsymbol{\tau}_{1}+\left(\boldsymbol{v} \cdot \boldsymbol{\tau}_{2}\right) \boldsymbol{\tau}_{2}$.

Consider now $\boldsymbol{\mu} \in H^{3 / 2}(\Gamma)$ such that $\boldsymbol{\mu} \cdot \boldsymbol{n}=0$. Because weighted Sobolev spaces satisfy the usual trace theorems, there exists $\boldsymbol{\varphi} \in W_{\alpha}^{2}(\Omega)$ such that $\boldsymbol{\varphi}=\boldsymbol{\mu}$ on $\Gamma$ and

$$
\|\boldsymbol{\varphi}\|_{W_{\alpha}^{2}(\Omega)} \leqslant\|\boldsymbol{\mu}\|_{H^{3 / 2}(\Gamma)} .
$$

Moreover, we can choose $\boldsymbol{\varphi}$ so that

$$
\nabla \boldsymbol{\varphi} \cdot \boldsymbol{n}=-\boldsymbol{n} \operatorname{div}_{\Gamma} \boldsymbol{\mu}_{\boldsymbol{\tau}}+\sum_{j=1}^{2}\left(\frac{\partial \boldsymbol{\mu}_{\boldsymbol{\tau}}}{\partial \ell_{j}} \times \boldsymbol{\tau}_{j}\right) \times \boldsymbol{n}
$$


With this choice and using the following relations

$$
\operatorname{div} \boldsymbol{\varphi}=\operatorname{div}_{\Gamma} \boldsymbol{\varphi}_{\boldsymbol{\tau}}+k_{\boldsymbol{\tau}}(\boldsymbol{\varphi} \cdot \boldsymbol{n})+(\nabla \boldsymbol{\varphi} \cdot \boldsymbol{n}) \cdot \boldsymbol{n} \quad \text { on } \quad \Gamma,
$$

where $k_{\boldsymbol{\tau}}$ is the principal curvature of $\Gamma$,

$$
\operatorname{curl} \boldsymbol{\varphi}=\sum_{j=1}^{2} \frac{\partial \boldsymbol{\varphi}_{\boldsymbol{\tau}}}{\partial \ell_{j}} \times \boldsymbol{\tau}_{j}+(\nabla \boldsymbol{\varphi} \cdot \boldsymbol{n}) \times \boldsymbol{n} \quad \text { on } \quad \Gamma
$$

and

$$
(\boldsymbol{\varphi} \times \boldsymbol{n}) \times \boldsymbol{n}=-\boldsymbol{\varphi}_{\boldsymbol{\tau}}
$$

we have

$$
\operatorname{div} \boldsymbol{\varphi}=0 \quad \text { and } \quad \operatorname{curl} \boldsymbol{\varphi}=\mathbf{0} \text { on } \Gamma,
$$

which implies that $\boldsymbol{\varphi} \in Z_{\alpha}(\Omega)$.

Next, if $\boldsymbol{u} \in \mathscr{D}(\bar{\Omega})$, then the Green formula (4.69) holds and, as a result, we can write

$$
\begin{aligned}
\left|\langle\operatorname{curl} \boldsymbol{u} \times \boldsymbol{n}, \boldsymbol{\mu}\rangle_{\Gamma}\right| & =\left|\langle\operatorname{curl} \boldsymbol{u} \times \boldsymbol{n}, \boldsymbol{\varphi}\rangle_{\Gamma}\right| \\
& \leqslant\|\boldsymbol{u}\|_{W_{-\alpha}^{0}(\Omega)}\|\boldsymbol{\varphi}\|_{W_{\alpha}^{2}(\Omega)}+\|\Delta \boldsymbol{u}\|_{\left(T_{\alpha}(\Omega)\right)^{\prime}}\|\boldsymbol{\varphi}\|_{T_{\alpha}(\Omega)} \\
& \leqslant\|\boldsymbol{u}\|_{H_{\alpha}(\Delta, \Omega)}\|\boldsymbol{\varphi}\|_{W_{\alpha}^{2}(\Omega)}
\end{aligned}
$$

which implies, thanks to (E.80), that

$$
\|\operatorname{curl} \boldsymbol{u} \times \boldsymbol{n}\|_{H^{-3 / 2}(\Gamma)} \leqslant\|\boldsymbol{u}\|_{H_{\alpha}(\Delta, \Omega)} .
$$

Therefore the linear mapping $\gamma:\left.\boldsymbol{u} \rightarrow \operatorname{curl} \boldsymbol{u}\right|_{\Gamma} \times \boldsymbol{n}$ defined on $\mathscr{D}(\bar{\Omega})$ is continuous for the norm of $H_{\alpha}(\Delta, \Omega)$. Since $\mathscr{D}(\bar{\Omega})$ is dense in $H_{\alpha}(\Delta, \Omega), \gamma$ can be extended by continuity to a mapping still called $\gamma$ defined on $H_{\alpha}(\Delta, \Omega)$ to $H^{-3 / 2}(\Gamma)$ and formula (4.69) holds for all $\boldsymbol{u} \in H_{\alpha}(\Delta, \Omega)$ and $\boldsymbol{\varphi} \in Z_{\alpha}(\Omega)$.

\section{References}

[1] H. Abboud, F. E. Chami, and T. Sayah. A priori and a posteriori estimates for three-dimensional Stokes equations with nonstandard boundary conditions. Numer. Methods Partial Differential Equations, 28(4):1178-1193, 2012.

[2] Y. Achdou, O. Pironneau, and F. Valentin. Effective boundary conditions for laminar flows over periodic rough boundaries. J. Comput. Phys., 147(1):187-218, 1998.

[3] C. Amrouche, V. Girault, and J. Giroire. Weighted Sobolev spaces for Laplace's equation in $\mathbb{R}^{n}$. J. Math. Pures Appl. (9), 73(6):579-606, 1994. 
[4] C. Amrouche, V. Girault, and J. Giroire. Dirichlet and Neumann exterior problems for the $n$ dimensional Laplace operator: an approach in weighted Sobolev spaces. J. Math. Pures Appl. (9), 76(1):55-81, 1997.

[5] C. Amrouche and M. Meslameni. Stokes problem with several types of boundary conditions in an exterior domain. Electron. J. Differential Equations, No. 196, 28 pp, 2013.

[6] C. Amrouche and N. H. Seloula. On the Stokes equations with the Navier-type boundary conditions. Differ. Equ. Appl., 3(4):581-607, 2011.

[7] S. K. Bhowmik, R. Belbaki, T. Z. Boulmezaoud, and S. Mziou. Solving two dimensional second order elliptic equations in exterior domains using the inverted finite elements method. Comput. Math. Appl., 72(9):2315-2333, 2016.

[8] T. Z. Boulmezaoud. Inverted finite elements: a new method for solving elliptic problems in unbounded domains. M2AN Math. Model. Numer. Anal., 39(1):109-145, 2005.

[9] T. Z. Boulmezaoud, K. Kaliche, and N. Kerdid. Inverted finite elements for div-curl systems in the whole space. Adv. Comput. Math., 43(6):1469-1489, 2017.

[10] T. Z. Boulmezaoud, S. Mziou, and T. Boudjedaa. Numerical approximation of second-order elliptic problems in unbounded domains. J. Sci. Comput., 60(2):295-312, 2014.

[11] J. H. Bramble and P. Lee. On variational formulations for the Stokes equations with nonstandard boundary conditions. RAIRO Modél. Math. Anal. Numér., 28(7):903-919, 1994.

[12] C. Conca, F. Murat, and O. Pironneau. The Stokes and Navier-Stokes equations with boundary conditions involving the pressure. Japan. J. Math. (N.S.), 20(2):279-318, 1994.

[13] C. Conca, C. Parés, O. Pironneau, and M. Thiriet. Navier-Stokes equations with imposed pressure and velocity fluxes. Internat. J. Numer. Methods Fluids, 20(4):267-287, 1995.

[14] H. Beirao da Veiga and F. Crispo. Sharp inviscid limit results under Navier slip boundary conditions. an $L^{p}$ theory. J. Math. Fluid Mech., 12(4):397-411, 2010.

[15] A. Dhifaoui, M. Meslameni, and U. Razafison. Weighted Hilbert spaces for the stationary exterior Stokes problem with Navier slip boundary conditions. J. Math. Anal. Appl., 472(2):1846-1871, 2019.

[16] V. Girault. The gradient, divergence, curl and Stokes operators in weighted Sobolev spaces of $\mathbb{R}^{3}$. J. Fac. Sci. Univ. Tokyo Sect. IA Math., 39(2):279-307, 1992.

[17] V. Girault. The Stokes problem and vector potential operator in three-dimensional exterior domains: an approach in weighted Sobolev spaces. Differential Integral Equations, 7(2):535-570, 1994. 
[18] J. Giroire. Étude de quelques problèmes aux limites extérieurs et résolution par équations intégrales. Thèse de Doctorat d'État. Université Pierre et Marie Curie, Paris, 1987.

[19] P. Grisvard. Elliptic problems in nonsmooth domains, volume 24 of Monographs and Studies in Mathematics. Pitman (Advanced Publishing Program), Boston, MA, 1985.

[20] B. Hanouzet. Espaces de Sobolev avec poids. Application au problème de Dirichlet dans un demi espace. Rend. Sem. Mat. Univ. Padova, 46:227-272, 1971.

[21] W. Jäger and A. Mikelić. On the roughness-induced effective boundary conditions for an incompressible viscous flow. J. Differential Equations, 170(1):96-122, 2001.

[22] A. Kufner. Weighted Sobolev spaces. A Wiley-Interscience Publication. John Wiley \& Sons Inc., New York, 1985.

[23] J.-L. Lions and E. Magenes. Problèmes aux limites non homogènes et applications. Vol. 1. Travaux et Recherches Mathématiques, No. 17. Dunod, Paris, 1968.

[24] H. Louati, M. Meslameni, and U. Razafison. Weighted $L^{p}$-theory for vector potential operators in threedimensional exterior domains. Math. Methods Appl. Sci., 39(8):1990-2010, 2016.

[25] M. Mitrea and S. Monniaux. The nonlinear Hodge-Navier-Stokes equations in Lipschitz domains. Differential Integral Equations, 22(3-4):339-356, 2009.

[26] C.L.M.H. Navier. Mémoire sur les Lois du Mouvement des fluides. Mem. Acad. Sci. Inst. de France (2), 6:389-440, 1827.

[27] C. Neto, D.R. Evans, E. Bonaccurso, H.J. Butt, and V. S. J. Craig. Boundary slip in newtonian liquids: a review of experimental studies. Rep. Prog. Phys., 68(12):2859-2897, oct 2005.

[28] J. Watanabe. On incompressible viscous fluid flows with slip boundary conditions. In Proceedings of the 6th Japan-China Joint Seminar on Numerical Mathematics (Tsukuba, 2002), volume 159, pages 161-172, 2003.

[29] Y. Xiao and Z. Xin. On the vanishing viscosity limit for the 3D Navier-Stokes equations with a slip boundary condition. Comm. Pure Appl. Math., 60(7):1027-1055, 2007. 